\title{
Die bundesdeutsche Wehrpflichtaussetzung durch Karl-Theodor zu Guttenberg. Alles eine Frage des Narrativs?
}

\author{
Timo Schummers
}

Online publiziert: 19. August 2020

(C) Der/die Autor(en) 2020

Zusammenfassung Die im Jahr 2010 vom damaligen Bundesminister der Verteidigung, Karl-Theodor zu Guttenberg (CSU), ergriffene Initiative zur Aussetzung der allgemeinen Wehrpflicht stellte eine Zäsur dar, die nur von den wenigsten Beobachtern zuvor erwartet worden war. Schließlich brach die ein Jahr später beschlossene Aussetzung des Wehrdienstes mit einer politisch-militärischen Kontinuitätslinie, die in der Bundesrepublik Deutschland seit 1956 Bestand hatte. Der vorliegende Beitrag wählt eine narrationsanalytische Perspektive, um zu untersuchen, inwiefern es zu Guttenberg durch die Verwendung zusammenhängender sprachlich-erzählerischer Muster gelang, das lange als politisches Tabuthema geltende Vorhaben schlussendlich als „Wehrrechtsänderungsgesetz 2011“ durchzusetzen. Ziel ist es, die erzählerischen Kontinuitäten, aber auch (überraschenden) Wendungen aufzuzeigen, die es ermöglichten, einen für die militärische Zukunft Deutschlands bedeutsamen Politikwechsel zu vollziehen. Dabei wird ersichtlich werden, dass zu Guttenberg für sein Vorhaben anfangs insbesondere finanzpolitische Argumente anführte, während später vor allem sicherheits- sowie gesellschaftspolitische Begründungsstrukturen bedeutungstragend wurden.

\section{The suspension of the German general conscription by Karl-Theodor zu Guttenberg. Is it all about narrative strategy?}

Abstract The in 2010 initiated suspension of the German general conscription by Karl-Theodor zu Guttenberg (CSU) was a political and military turning point in the Federal Republic of Germany, which only a few people had anticipated. Moreover, the therewith coupled political change could only partially be explained in political

\footnotetext{
T. Schummers $(\triangle)$

Campus Landau, Institut für Germanistik, Universität Koblenz-Landau,

Fortstraße 7, 76829 Landau in der Pfalz, Deutschland

E-Mail: schummers@uni-landau.de
} 
science so far. This is why the article chooses a rather new perspective by using an analytical approach, that has been established in political science in the last few years and focalizes a narrative analysis of the case study. It will be shown that Karl-Theodor zu Guttenberg used some coherent narrative patterns just like (unexpected) twists, which helped him to fulfill his political project in 2011. While he first spoke primarily about financial issues, he later on used arguments, which especially focused aspects of security policy and socio-politically reasons.

\section{Einleitung}

Eine Beschäftigung mit Erzählungen in der Politik hat in der deutschsprachigen Politikwissenschaft erst seit wenigen Jahren Konjunktur. Im Zuge dessen hat sich eine politikwissenschaftliche Strömung herausgebildet, die an die noch junge Tradition konstruktivistischer bzw. post-positivistischer Forschungsansätze (vgl. z.B. Gottweis 2003) und damit der interpretativen Policy-Forschung (vgl. z. B. Münch 2016) anknüpft. Innerhalb dieses Forschungsfeldes werden politische Narrative als eigenständige Analyseobjekte betrachtet (vgl. z. B. Gadinger et al. 2014a). Grundlegend hierfür ist die Annahme, dass „Erzählungen [...] Politik unmittelbar herstellen“ (Jarzebski 2015, S. 374), sodass das Erzählen als „ein Kernmoment der sprachlichen Konstruktion von Sachverhalten“" (ebd.) gilt.

Die politikwissenschaftliche Erzählforschung bietet eine Alternative zu den bislang gängigen, oftmals rationalistischen Ansätzen der Politikfeldanalyse und wird daher als Gewinn für eine moderne Regierungsforschung angesehen (vgl. Gadinger et al. 2014b, S. 27; Florack et al. 2015, S. 626). Sie ermöglicht eine erweiterte Perspektive auf politische Phänomene, womit sie insbesondere dann einen fruchtbaren Zugang bereitstellt, wenn die klassische Policy-Forschung weniger erschöpfende Ausdeutungen für politische Problemlösungsprozesse entwickeln kann.

Bislang konnte die im Jahr 2011 vom ehemaligen Bundesminister der Verteidigung, Karl-Theodor zu Guttenberg (CSU), initiierte Aussetzung der bundesdeutschen Wehrpflicht zwar mit unterschiedlichen Deutungen versehen, jedoch nicht gänzlich erklärt werden. Diese wird heute beispielsweise im Feld der deutschen Außenpolitikforschung rückblickend als ,rapide[r], wenn nicht gar rabiate[r] Politikwechsel" (Harnisch und Weiß 2014, S. 232) bezeichnet, der mit einer jahrzehntelang existierenden politischen Kontinuitätslinie in einem allerdings längst reformbedürftigen Politikfeld brach. Doch obwohl der Wandel des deutschen Wehrsystems umfangreich untersucht ist, überzeugen bislang größtenteils angeführte rational-strukturalistische Erklärungen nicht vollends (vgl. auch ebd., S. 216). Denn ein Ende des Wehrdienstes stellte noch bis zum Frühjahr 2010 ein politisches Tabuthema dar (vgl. z. B. Lohse und Wehner 2011, S. 316; Hartleb 2012, S. 35). Zwar wurden solche Überlegungen unter anderem durch die FDP und Bündnis 90/Die Grünen seit Beginn der 2000er-Jahre verstärkt geäußert, allerdings galten diese seither vor allem in der CDU/CSU ,,als wirklichkeitsferne Wünsche von Klientelparteien“ und wurden ,zuverlässig [...] abgewiesen“ (beide Zitate: Lohse und Wehner 2011, S. 316). Der Grund dafür lag in der historisch-kulturellen Prägekraft der 1956 wesentlich auf Initiative der Unionsparteien eingeführten allgemeinen Wehrpflicht (vgl. CDU 
Deutschland 2010b, S. 1; Lohse und Wehner 2011, S. 311), die nach der Einsetzung ein zentrales Instrument der Verbindung zwischen Streitkräften, Bevölkerung und Staat wurde (vgl. z. B. von Bredow 2015, S. 209). Zudem war der Wehrdienst in der ein Jahr zuvor gegründeten Bundeswehr eine für die Wehrdienstleistenden wichtige Sozialisationsinstanz und galt als ,Symbol der Einsatzbereitschaft für den freiheitlichen Verfassungsstaat“" (Steinbach 2011, S. 10). Um die Wehrpflicht - nicht zuletzt ein Teil des konservativen Markenkerns der CDU/CSU - vor diesem Hintergrund erfolgreich auszusetzen, musste zu Guttenberg in seinem politischen Umfeld - und insbesondere innerhalb der Union - große Überzeugungsarbeit leisten, weshalb aus einem narrationsanalytischen Blickwinkel heraus das Fallbeispiel von besonderem Untersuchungsinteresse ist.

In diesem Beitrag soll die Annahme vertreten werden, dass der (rasche) Erfolg des ehemaligen Verteidigungsministers bei seinem Vorhaben zur Wehrpflichtaussetzung vornehmlich in seinem hierbei verwendeten Narrativ begründet liegt. Um einen abrupten Politikwechsel vollziehen zu können, hat Karl-Theodor zu Guttenberg - so die untersuchungsleitende These - eine sinngenerierende Erzählung entfaltet, die in ihrer Struktur sowie Wirkkraft mithilfe einer politikwissenschaftlichen Narrativanalyse im Folgenden näher untersucht wird. Im Zentrum steht die Frage, auf welche Art und Weise zu Guttenberg die Wehrpflichtaussetzung durch die Konstruktion spezifischer Narrativmuster zu legitimieren versuchte. Außerdem ist zu betrachten, welche Muster die verwendeten Erzählstrukturen aufwiesen, sodass diese politische Zustimmung für den Policy-Wandel erzeugen und bis dahin bestehende diskursive Argumentationsstrukturen zur Aufrechterhaltung der Wehrpflicht öffentlichkeitswirksam in den Hintergrund drängen konnten.

Um diese Fragen zu beantworten, wird der Beitrag zunächst die theoretischen sowie methodischen Grundlagen des ausgewählten narrationsanalytischen Forschungszugangs aufzeigen. Sodann werden die durch Karl-Theodor zu Guttenberg verwendeten Erzählmuster bei seinem Vorhaben zur Aussetzung der bundesdeutschen Wehrpflicht untersucht. ${ }^{1}$ Dabei wird festzustellen sein, dass sich die narrativen Strukturen im Laufe der Zeit veränderten und nach anfänglich finanzpolitischen Argumenten vor allem sicherheits- sowie gesellschaftspolitische Begründungen ins Zentrum des zu analysierenden Narrativs rückten. Insgesamt folgt der Aufsatz einer qualitativinterpretativen Forschungsausrichtung, die darauf abzielt, den von zu Guttenberg initiierten Politikwechsel nachzuvollziehen und eine zu den bisherigen Erklärungen divergierende Deutung der Geschehnisse anzubieten.

\footnotetext{
1 Es sei bereits an dieser Stelle darauf hingewiesen, dass auf eine Zusammenfassung der nach Einsetzung der Wehrpflicht im Jahr 1956 bis zum Aufkommen der Erwägungen zur Wehrpflichtaussetzung im Jahr 2010 dominierenden diskursrelevanten Narrativ- sowie Diskussionsstränge zur Wehrpflicht-Thematik in diesem Beitrag größtenteils verzichtet werden muss. Für verschiedene Perspektiven auf diese Zeitspanne sowie insbesondere auf die drei Gründungsnarrative der Bundeswehr als Parlamentsarmee mit Staatsbürgern in Uniform und dem Konzept der Inneren Führung sei stattdessen exemplarisch auf die Darstellungen bei Seidler und Reindl (vgl. 1973), Raidel (vgl. 1998), Groß und Lutz (vgl. 1998), Steinbach (vgl. 2011) sowie von Bredow (vgl. 2015) verwiesen.
} 


\section{Der Forschungszugang Politikwissenschaftliche Narrativanalyse}

Lange Zeit galten in der Forschung Erzählungen als genuine Untersuchungsobjekte der Literaturwissenschaft und als Gegenstände der literaturwissenschaftlichen Erzähltheorie (vgl. Gadinger et al. 2014b, S. 3). ${ }^{2}$ Diese Sichtweise änderte sich jedoch nach dem innerhalb der Policy-Forschung vollzogenen argumentative turn bzw. der dort seit Mitte der 1990er-Jahre postulierten interpretativen Wende (vgl. z.B. Barbehön und Münch 2014, S. 149). Seitdem interessiert sich auch jenseits der literaturwissenschaftlichen Disziplinen die Politikwissenschaft für eine Analyse von (politischen) Erzählungen, sodass diese als Untersuchungsgegenstand vermehrt Berücksichtigung finden.

\subsection{Entstehungslinien und theoretisch-konzeptionelle Grundlagen der aktuellen politikwissenschaftlichen Narrativforschung}

Den Ausgangspunkt für heutige Narrativanalysen bilden Ansätze aus dem englischsprachigen Raum, die zeitgleich mit dem sich vollziehenden interpretativen Paradigmenwechsel am Ende der 1990er-Jahre in die Policy-Forschung eingebracht wurden (vgl. z.B. Stone 1989; Kaplan 1993; Roe 1994). All diese Ansätze vereint der Anspruch, mit je unterschiedlichen Vorgehensweisen zusammenhängende Argumentations- und Erzählmuster bei politischen Problembearbeitungsprozessen nachvollziehen zu wollen (vgl. Schneider und Janning 2006, S. 173-174; van Eeten 2007, S. 251-255).

Seit den 2000er-Jahren lässt sich nun ebenfalls in Deutschland eine verstärkte Hinwendung zur Beschäftigung mit Narrativen in der Politik konstatieren. Inspiriert von wissenssoziologischen Diskursanalysen (vgl. z. B. Viehöver 2008, 2011, 2013) sind innerhalb der Politikwissenschaft in den letzten Jahren umfangreiche Zugänge zu politischen Erzählungen entstanden (vgl. z. B. Gadinger et al. 2014a; Hofmann et al. 2014; Müller und Precht 2019; zuletzt Yildiz 2020). Gerade die innerhalb der deutschen Policy-Forschung durch eine Autorengruppe um Frank Gadinger, Taylan Yildiz und Sebastian Jarzebski ${ }^{3}$ entwickelten konzeptionellen Ansätze für politikwissenschaftliche Narrativanalysen sind in diesem Zusammenhang herauszuheben. Sie plädieren für akteurzentrierte Untersuchungen und eine „Wiederentdeckung des Akteurs, der im Rahmen der Narrativanalyse als Erzähler analytisch aufgewertet wird“" (Jarzebski 2015, S. 380). Unter Berücksichtigung dieser Prämisse sind in den letzten Jahren zahlreiche fruchtbare Beiträge veröffentlicht worden, die innerhalb eines ausgewählten Politikfeldes narrativ-eingesetzte Sprachmittel analysieren (vgl.

\footnotetext{
${ }^{2}$ Für eine Zusammenfassung der zentralen Annahmen und wesentlichen Bestandteile der literaturwissenschaftlichen Erzähltheorie (Narratologie) sowie deren Implikationen für politikwissenschaftliche Untersuchungen siehe die kurzen Ausführungen bei van Eeten (vgl. 2007, S. 252-253). Die „Grundzüge einer Allgemeinen Erzähltheorie“ in aller Ausführlichkeit stellt darüber hinaus Koschorke (2013) zusammen.

3 Die drei Politikwissenschaftler waren Teil eines an der NRW School of Governance in Kooperation mit dem Käte Hamburger Kolleg/Centre for Global Cooperation Research (beide Universität Duisburg-Essen) initiierten Forschungsprojektes mit dem Schwerpunkt Politische Narrative (vgl. Gadinger et al. 2014b, S. 5, FN 1).
} 
z. B. Gadinger et al. 2014c, 2019; Jarzebski 2015; Gadinger 2019; Gadinger und Simon 2019).

Die neuen Arbeiten können dabei gewissermaßen als Antwort auf bis dahin in diesem politikwissenschaftlichen Themenfeld festgestellte Forschungslücken (vgl. z. B. Biegoń und Nullmeier 2014, S. 60-61) verstanden werden. Denn Gadinger et al. zielen darauf $\mathrm{ab}$, forschungstheoretisch umfassende ,Möglichkeiten einer politikwissenschaftlichen Narrativanalyse" (Gadinger et al. 2014c, S. 67) auszuloten. Zugleich sieht ihr qualitativ-interpretativ ausgearbeiteter und in konstruktivistischer Tradition stehender Ansatz vor, analytisch-konzeptionelle Vorschläge für die Untersuchung von politischen Narrationen anzubieten (vgl. z. B. Yildiz et al. 2015, S. 426-428; Gadinger et al. 2019, S. 91-95).

Einen wichtigen Ausgangspunkt zur Herleitung der Relevanz einer politikwissenschaftlichen Erzählforschung entlehnt die Autorengruppe aus dem zum Ende des 20. Jahrhunderts in den Geisteswissenschaften vollzogenen linguistic turn. Dieser bildete die heute auch in der Politikwissenschaft zunehmend anerkannte Auffassung eines zentralen Zusammenhangs von Sprache und (Welt-)Verstehen heraus, mithin das Postulat, dass Denken und die Wahrnehmung von Wirklichkeit nicht außerhalb des Mediums Sprache möglich ist (vgl. Stierstorfer 2013, S. 447-448; Gadinger et al. 2014b, S. 3-4). Jenseits dessen ist allerdings der für die Erzählforschung konstitutive Narrativ-Begriff keinesfalls eindeutig zu bestimmen. Vielmehr lässt sich insbesondere im Vergleich zu ähnlichen Begriffen wie Geschichte, Erzählung und Narration keine Definition finden, die sich allgemein etabliert hätte (vgl. Gadinger et al. 2014c, S. 70, FN 2). In Anlehnung an bereits durchgeführte politikwissenschaftliche Narrativanalysen (vgl. z. B. Jarzebski 2015, S. 368, FN 2) werden deshalb in diesem Beitrag die Begriffe Geschichte, Erzählung, Narration und Narrativ synonym verwendet. Zur definitorischen Annäherung an den NarrativBegriff seien dennoch wichtige strukturell-inhaltliche Bedeutungsgehalte genannt.

So wird hier ein Narrativ verstanden als „Erzählung einer Sequenz von Ereignissen“ (Barbehön und Münch 2014, S. 153), bei welcher Akteure durch die Nutzung narrativer Techniken verschiedene (Sinn-)Elemente und (Wissens-)Bestandteile reduktiv in einer komplexen räumlichen sowie kausalen Ordnung erzählerischkohärent in Bezug setzen und in temporalen Mustern miteinander verknüpfen. Diese drei Merkmale gemeinsam lassen - eingebettet in einen gesellschaftspolitischkulturellen Kontext - durch eine komplexitätsreduzierende Synthese ihrer Ausgestaltung in konfigurierten Plots einen narrativen Ereigniszusammenhang dynamisch entstehen, sodass als jene Plots sequenziell organisierte Erzählmuster als zentrale Strukturmerkmale von (politischen) Narrativen anzusehen sind. Außerdem sorgen diese Eigenschaften dafür, dass narrative Verknüpfungsleistungen darüber entscheiden, inwiefern ein politisches Problem (öffentlich) wahrgenommen wird und zur Bearbeitung auf die Agenda der Politik rückt (vgl. ebd., S. 152-153; Gadinger et al. 2014b, S. 25, 2014c, S. 72-75; Viehöver 2014, S. 78-79, 85-87; Jarzebski 2015, S. 373, 380; Yildiz et al. 2015, S. 427).

Als weiterer wichtiger konzeptioneller Eckpunkt der Überlegungen nach Gadinger et al. ist das zugrundeliegende Akteurverständnis hervorzuheben. Denn dieses stellt die (welt-)erzählerischen Fähigkeiten eines politisch Handelnden (als homo 
narrans ${ }^{4}$ ) in den Mittelpunkt. Jener wiederum wirkt im Rahmen gesellschaftspolitischer Umweltbedingungen und Handlungskorridore durch die sinngenerierende Verwendung von Narrativen unmittelbar an der Herstellung von Politik bzw. dort ausgestalteter Problemlösungen mit (vgl. Gadinger et al. 2014b, S. 5, 23, 2014c, S. 69, 78; Jarzebski 2015, S. 371, 380, 382).

Um - in einem Kontext demokratischer Aushandlungsprozesse - die zusammenlaufenden Prozesse schließlich angemessen untersuchen zu können, wird das „Erzählen als politische Praktik“ (Gadinger et al. 2014b, S. 9) begriffen. Dies lenkt den Analysefokus auf die narrativ-kommunikativen Kompetenzen eines erzählenden Akteurs und führt dazu, Erzählungen im Hinblick auf ihre Plausibilisierungskräfte bei dem Versuch zur Generierung politischer Legitimität zu untersuchen sowie deren inhärente machtpolitische Deutungsansprüche offenzulegen. Außerdem ist in einer dritten Perspektive auf die Ausprägungen politischer Polyphonie zu achten. Damit sind durch das gesellschaftspolitische Miteinander verschiedener Erzähler und Zuhörer im politischen Prozess grundsätzlich anzunehmende narrative Eigendynamiken und häufig vielstimmige Erscheinungsweisen von Narrationen gemeint, die von den Fragen nach Legitimität und Macht stark beeinflusst werden (vgl. Gadinger et al. 2014b, S. 10-13, 2014c, S. 71-72, 87; Gadinger und Yildiz 2017, S. 159-161).

\subsection{Methodik der politikwissenschaftlich-empirischen Narrativanalysen}

Als Untersuchungsraster zur Analyse politischer Erzählungen und ihrer Muster wird von Gadinger et al. eine kategoriale Dreiteilung vorgeschlagen. Aufgegliedert werden die Erzählelemente (Leit-)Metaphern, Rollen und Plots, deren „Rekonstruktion stichhaltige Anhaltspunkte für die kollektive Strukturierung politischer Möglichkeitsräume liefert“ (Gadinger und Yildiz 2017, S. 164). Somit steht am Beginn eines jeden Forschungsprozesses zunächst die Identifikation von wichtigen sprachlichen (Leit-)Metaphern, welche eine kognitive, normative und dialogische Dimension besitzen (vgl. z. B. Gadinger et al. 2015, S. 15, 2019, S. 96; Yildiz et al. 2015, S. 426).

Ihre erste, kognitive Funktion entfalten die (Leit-)Metaphern in ihrer Fähigkeit, komplexe sowie sinnlich nicht direkt erfahrbare Sachverhalte - durch ausgewählte bildlich-metaphorische Übertragungen eines Herkunftsbereichs auf einen Zielbereich - in der Sphäre der Politik unmittelbar verständlich zu machen. Sie werden in Anlehnung an die Konzeptualisierungstheorie der Metapher nach George Lakoff und Mark Johnson (vgl. z. B. 2008) als mentale Konzepte verstanden, die menschliches Verstehen und Wahrnehmen prägen. Außerdem können sie im Feld der Politik Handlungsoptionen vorschlagen und bereitstellen. Neben der kognitiven Prägekraft ist so ihre normative Eigenschaft angesprochen, da Metaphern durch Zustands- und Deutungsschilderungen mittels der Suggestion auf die politische Willensbildung einwirken. Ein zuletzt charakteristisches dialogisches Merkmal entsteht aufgrund der ,interpretativen Offenheit von Metaphern“ (Gadinger et al. 2015, S. 15). Denn durch eine nie vollständig abgeschlossene Bedeutungsfestlegung können diese in einer politisch-polyphonen Diskussion hinsichtlich ihrer wirklichkeitsgenerierenden

\footnotetext{
4 Zu diesem, vor allem in den 1980er Jahren innerhalb der Kommunikationswissenschaften entwickelten Konzept siehe beispielsweise Fisher (vgl. 1985) oder zusammenfassend Koschorke (vgl. 2013, S. 9-12).
} 
Perspektivierung verändert werden. Ebenso sind sie in der Lage, politische Kontroversen zu strukturieren (vgl. Gadinger et al. 2014c, S. 76, 2015, S. 15; Jarzebski 2015, S. 372; Yildiz et al. 2015, S. 426-427; Gadinger 2019, S. 127-128).

Aufgrund dieser bedeutenden Funktionen scheint es allerdings geboten, bei politischen Narrativanalysen einen definitorisch insgesamt weit gefassten MetaphernBegriff anzulegen. Denn da (Leit-)Metaphern ,den kollektiven Austausch darüber strukturieren können, was der Fall ist“" (Yildiz et al. 2018, S. 142), muss es bei zu untersuchenden Policy-Fallbeispielen darum gehen, jene ,,semantischen Stellen“ aufzudecken, ,die Auskunft über die basale Konstruktion der Ereignisse geben“ (beide Zitate: ebd.). Die narrativanalytische Komponente (Leit-)Metaphern sollte deshalb eine Ermittlung all derjenigen Schlüsselwörter bzw. Schlüsselkonzepte umfassen, die vonseiten politischer Akteure zur Gestaltung von Politik eingesetzt werden.

Neben der Identifizierung dieser sprachlichen Bausteine sind fernerhin auffindbare Rollenzuschreibungen (Rollen) zu erhellen, die im Handlungsfeld der Politik herausgebildet und in eine politische Erzählung eingeflochten werden (vgl. z. B. Gadinger et al. 2015, S. 15). Eine Analyse von Rollenkonstrukten ermöglicht es, in politischen Erzählungen , die zentralen Protagonisten zu identifizieren und [...] eine differenzierte Sicht auf die Akteurskonstellationen [...] zu erhalten“ (Yildiz et al. 2018, S. 142-143). Unterscheiden lassen sich diese Rollen in Sender und Empfänger, Held und Bösewicht (Anti-Held) sowie Objekt und Helfer (vgl. z. B. Viehöver 2008, S. 246, 2011, S. 213). Auch andere Zuschreibungen wie eine Differenzierung von Helden und Schurken, Intriganten oder Verrätern sowie Verschwörern oder Rettern lassen sich in politischen Erzählungen wiederfinden (vgl. Yildiz et al. 2015, S. 427; Gadinger 2019, S. 128).

Schlussendlich entwickeln sich aus der Gleichzeitigkeit von zu entschlüsselnden (Leit-)Metaphern und Akteurskonstellationen jeweils mit einer kausalen sowie zeitlichen Ordnung versehene Plots (Emplotments). Diese lassen dann in Form sequenziell organisierter Erzählmuster einen narrativen Ereigniszusammenhang in einem politischen (Gesamt-)Narrativ entstehen (vgl. Jarzebski 2015, S. 377; Yildiz et al. 2015, S. 427, 2018, S. 143; Gadinger 2019, S. 129; siehe auch Abschn. 2.1).

Alles in allem müssen bei einer politikwissenschaftlichen Narrativanalyse die aufgezeigten Bausteine (siehe zusammenfassend auch Tab. 1) beleuchtet werden, um die von narrativ tätigen Akteuren mit dem Ziel der Herstellung von Dringlichkeitsansprüchen und Handlungslegitimation präsentierten Geschichten im Feld des Politischen detailliert erfassen zu können. Angemerkt sei, dass die vorgenommene kategoriale Dekonstruktion der - oftmals fluiden - politischen Erzählungen und ihrer Muster allein analytisch zu begründen ist. ${ }^{5}$ Erst diese konzeptionelle Ausdifferenzierung ermöglicht eine systematische Narrationsanalyse von (innenpolitischen) PolicyBeispielen, um zu verdeutlichen, dass Erzählungen die politische Realität weitaus stärker beeinflussen, als dies bislang (an-)erkannt wurde (vgl. auch Jarzebski 2015, S. 382).

\footnotetext{
5 Dies bedingt, dass das dargestellte Analyseraster als Heuristik zu verstehen ist und durch begründete forschungstheoretische Herleitungen auch anders strukturiert sein könnte. Siehe beispielweise für methodisch abweichende Analysen die Arbeiten von Oppermann und Spencer (vgl. 2016, 2018) sowie Spencer (vgl. 2016, 2019) im Feld der Internationalen Beziehungen.
} 
Tab. 1 Methodische Dimensionen einer akteurzentrierten Untersuchung politischer Erzählungen und ihrer Muster unter Berücksichtigung von Vielstimmigkeit (Polyphonie)

\begin{tabular}{|c|c|}
\hline $\begin{array}{l}\text { Methodische } \\
\text { Dimension }\end{array}$ & Operationalisierter Zugriff \\
\hline $\begin{array}{l}\text { (Leit-)Me- } \\
\text { taphern }\end{array}$ & $\begin{array}{l}\text { Akteurzentrierte Untersuchung narrativ verwendeter sprachlich-metaphorischer Aus- } \\
\text { drücke/Schlüsselwörter/Schlüsselkonzepte im Hinblick auf die dadurch entstehenden } \\
\text { kognitiven, normativen sowie dialogischen Kräfte }\end{array}$ \\
\hline Rollen & $\begin{array}{l}\text { Akteurzentrierte Untersuchung von fallbeispielbezogenen Rollenzuschreibungen/ } \\
\text { Rollenkonstrukten/Akteurskonstellationen }\end{array}$ \\
\hline Plots & $\begin{array}{l}\text { Akteurzentrierte Untersuchung narrativ-sequenziell durch die Gleichzeitigkeit verwen- } \\
\text { deter (Leit-)Metaphern und Akteurskonstellationen in einer spezifischen Ordnung rhe- } \\
\text { torisch-argumentativ verknüpfter Sachverhalte zur bedeutungskonstituierenden Identifi- } \\
\text { zierung politischer Probleme und zustimmungsgenerierenden Legitimierung politischer } \\
\text { Lösungsvorschläge }\end{array}$ \\
\hline
\end{tabular}

Quelle: Eigene Darstellung auf der Basis der vorherigen Erläuterungen.

\section{Analyse des Wehrpflichtaussetzungsnarrativs Karl-Theodor zu Guttenbergs}

Die folgende Narrativanalyse nimmt die Zeit Karl-Theodor zu Guttenbergs als Bundesminister der Verteidigung zwischen dem 28. Oktober 2009 und dem 03. März 2011 in der 17. Wahlperiode der Bundesrepublik Deutschland (vgl. Löwenstein und Schäffer 2011; Spiegel Online 2011) näher in den Blick. Denn als politischer Hauptakteur war zu Guttenberg maßgeblich für die Aussetzung der bundesdeutschen Wehrpflicht - die am 24. März 2011 durch den Bundestag und am 15. April 2011 durch den Bundesrat verabschiedet wurde (vgl. ZEIT Online 2011a; Kulak et al. 2016, S. 17) - verantwortlich. Zwar musste sich der damalige Minister gegen einige Widerstände durchsetzen, trotzdem gelang es ihm, im Jahr 2010 eine Bundeswehrreform anzustoßen, die im Kern die Aussetzung des Wehrdienstes zum 01. Juli 2011 vorsah.

\subsection{Vorgehensweise der Untersuchung}

Im Folgenden dieses Beitrags soll eine interpretativ-explorative Vorgehensweise gewählt werden, auf deren Grundlage ein auf systematisch ausgewähltes Textmaterial basierender Korpus rekonstruktiv sowie additiv ausgewertet wird. Dieser enthält die wichtigsten Reden Karl-Theodor zu Guttenbergs in seiner Amtszeit als Bundesverteidigungsminister mit Bezug zum Thema Aussetzung der Wehrpflicht. Analysiert werden in einem Zeitraum zwischen dem 20. Januar 2010 und dem 24. Februar 2011 einerseits alle themenbezogenen Reden zu Guttenbergs im Deutschen Bundestag (vgl. Deutscher Bundestag 2010a, 2010b, 2010c, 2010d, 2010e, 2010f, 2011; siehe auch Tab. 2 im Anhang) sowie andererseits ausgesuchte öffentlichkeitswirksame Reden außerhalb des Bundestages, in welchen er sich ausführlicher mit der 
Wehrpflicht-Thematik auseinandersetzte (vgl. BMVg 2010a, 2010b; Bundespresseamt 2010; CDU Deutschland 2010a; siehe auch Tab. 3 im Anhang). ${ }^{6}$

Insgesamt wird damit der Analyseschwerpunkt vor allem auf einer akteurzentrierten Untersuchung der durch den ehemaligen Verteidigungsminister vor dem Deutschen Bundestag verwendeten Narrativmuster liegen, wobei ebenso zwei Reden vor Vertretern der Bundeswehr sowie jeweils eine Rede vor der bayerischen Frauenunion ${ }^{7}$ und auf dem CDU-Parteitag $2010 \mathrm{zu}$ betrachten sind. Diese Zusammenstellung ist darauf zurückzuführen, dass sich - wie hier vermutet wird - die erzählerischen Muster zu Guttenbergs vornehmlich in seinen Reden im Parlament sowie in ausgewählten Reden außerhalb des Bundestages wiederfinden lassen, welche auf diese Weise in der Öffentlichkeit wirken und einen großen Einfluss auf die von ihm konstruierte (politische) Realität entfalten konnten. Zudem wird es mit der getroffenen Auswahl möglich, sowohl die in der parlamentarischen als auch die in der bundeswehrinternen und parteipolitischen (Rede-)Arena verwendeten Erzählbestandteile aufzudecken.

Die Narrativanalyse soll von der These getragen werden, dass zu Guttenberg als entscheidender Akteur erstmalig nach schon in den 1990er-Jahren begonnenen Reformdiskussionen zur bundesdeutschen Wehrpflicht (siehe dazu auch die Literaturverweise in FN 1) maßgeblich durch die Nutzung von noch zu identifizierenden (Leit-)Metaphern sowie Rollen und hierdurch entstehenden Plots eine überzeugende (Gesamt-)Erzählung entwickeln und mit dieser die Wehrdienstaussetzung politisch durchsetzen konnte.

Unter Berücksichtigung des vorgestellten Korpus wird als zeitlicher Startpunkt für die Narrativrekonstruktion der 26. Mai 2010 gewählt. An diesem Tag hielt der erst wenige Monate zuvor als Bundesminister der Verteidigung vereidigte Karl-Theodor zu Guttenberg anlässlich eines Besuchs der Führungsakademie der Bundeswehr und der Kommandeurtagung der Streitkräftebasis in Hamburg seine Grundsatzrede, welche als „wichtigste Rede seines ersten Amtsjahres“ (Lohse und Wehner 2011, S. 299) wahrgenommen wurde. Sie löst zur damaligen Zeit einen „Paukenschlag““ aus, denn was Guttenberg dort verkündet, erscheint als ,eine Sensation: der Anfang vom Ende der Wehrpflicht“" (beide Zitate: ebd.).

\footnotetext{
${ }^{6}$ Reden, die der damalige Minister zu Geschehnissen in laufenden Auslandseinsätzen der Bundeswehr oder auf Gast- sowie Gedenkveranstaltungen hielt, werden entsprechend nicht berücksichtigt.

7 Diese Rede anlässlich der Fachkonferenz Zukunft Bundeswehr - eine gesamtgesellschaftliche Herausforderung (vgl. BMVg 2010b) wurde exemplarisch ausgewählt, da zu Guttenberg die darin zu findenden Argumentationsstrukturen - wie Lohse und Wehner (vgl. 2011, S. 336, 341) schildern - ab September 2010 mehrmals vor einer interessierten Öffentlichkeit, vor allem aber einem unionszugewandten Publikum entfaltet habe. Für eine Manuskriptbereitstellung dieser Rede sowie der Hamburger Grundsatzrede zu Guttenbergs (vgl. BMVg 2010a) sei an dieser Stelle Herrn Oberstleutnant und Pressestabsoffizier Dr. Harald Fritz Potempa am Zentrum für Militärgeschichte und Sozialwissenschaften der Bundeswehr in Potsdam gedankt, da beide Redemanuskripte nicht mehr anderweitig zugänglich waren.
} 


\subsection{Inhaltliche Bestandteile des Wehrpflichtaussetzungsnarrativs zu Guttenbergs}

Wenngleich sich die ersten Andeutungen zu Guttenbergs hinsichtlich möglicher Pläne einer Wehrdienstaussetzung rückblickend tatsächlich vorrangig auf den benannten Tag im Mai 2010 zurückführen lassen (vgl. auch Meyer 2010, S. 19; Harnisch und Weiß 2014, S. 226-227), so war das Thema Wehrpflicht schon seit längerem Gegenstand politischer Diskussionen. Es war vor allem die FDP, die nach der Bundestagswahl vom 27. September 2009 als Bedingung für eine erfolgreiche Koalitionsbildung mit der CDU/CSU schon frühzeitig eine Aussetzung des bundesdeutschen Wehrdienstes forderte (vgl. Kulak et al. 2016, S. 5) ${ }^{8}$ Diesem Anliegen wollte die Union jedoch zunächst nicht folgen. Stattdessen einigten sich die späteren Koalitionsparteien auf einen Kompromiss (vgl. ebd.), der laut Koalitionsvertrag vorsah, dass CDU, CSU und FDP zwar „,im Grundsatz an der allgemeinen Wehrpflicht“ festhalten, zugleich aber anstreben, ,die Wehrdienstzeit bis zum 1. Januar 2011 auf sechs Monate zu reduzieren“ (beide Zitate: CDU et al. 2009, S. 124). Ebenso wurde die Einsetzung einer Kommission vereinbart, ,die [...] Eckpunkte einer neuen Organisationsstruktur der Bundeswehr [...] zu erarbeiten hat" (ebd.).

Bevor Bundesverteidigungsminister Karl-Theodor zu Guttenberg diese Kommission am 12. April 2010 schließlich einsetzte (vgl. Meyer 2010, S. 2), hatten die Vorbereitungen zur Umsetzung der Wehrdienstverkürzung aber bereits begonnen. Das Vorhaben nahm vor allem mit dem Beschluss des Bundeskabinetts vom 19. Mai 2010 seine vorläufig letzte Hürde (vgl. Deutscher Bundestag 2010b, S. 4194, 2010c, S. 4815). Am gleichen Tag - und damit nur eine Woche vor seinem Auftritt in Hamburg - betonte der Minister im Bundestag jedoch noch, dass die Entscheidung ,nicht als Einstieg in den Ausstieg aus der Wehrpflicht missverstanden werden darf“" (Deutscher Bundestag 2010b, S. 4195). Dass die Wehrpflichtaussetzung dann Anfang des Jahres 2011 von zu Guttenberg als ,einer der Kernpunkte der Neuausrichtung der Bundeswehr“ (Deutscher Bundestag 2011, S. 10424) verkündet werden wird, konnte zu diesem Zeitpunkt keiner ahnen. Mitnichten war diese Entscheidung vorgezeichnet (vgl. auch Lohse und Wehner 2011, S. 307; Harnisch und Weiß 2014, S. 225-226; Kulak et al. 2016, S. 2).

\subsubsection{Narratives Muster I: Finanz- und haushaltspolitischer Plot}

Dass die Ende Mai 2010 in Hamburg gehaltene Grundsatzrede Karl-Theodor zu Guttenbergs eine politische Überraschung bereithalten sollte, klingt bereits zu Beginn seines Auftritts an. So erklärt der Minister gleich nach der Begrüßung, dass neben seiner Absicht, über Grundsätzliches sprechen zu wollen - noch eine Passage kurzfristig mit hinzugekommen sei, ,[e]in Entschluss[,] der vielleicht nicht leicht fällt, weil wir in diesen Tagen mit Zahlen zu hantieren haben, mit denen die Bun-

\footnotetext{
8 Bei der Bundestagswahl 2009 hatte die FDP 14,6\% der Zweitstimmen erhalten. Mit diesem, bislang besten Wahlergebnis ihrer Parteigeschichte gingen die Freien Demokraten daher selbstbewusst in die Koalitionsverhandlungen mit der Union, die 33,8\% der Zweitstimmen verbuchen konnte (vgl. Der Bundeswahlleiter 2020).
} 
desregierung in den letzten Jahren so noch nicht konfrontiert war" (BMVg 2010a, S. 1). Seine Beschreibung, zu diesem ergänzenden Teil der Rede habe er sich ,,heute entschlossen“ (ebd.), ist als erster Bestandteil seines sich entwickelnden, ersten narrativen Musters zu sehen, denn die folgenden Ausführungen führen systematisch auf die als spontan deklarierte Passage hin (vgl. auch Lohse und Wehner 2011, S. 299). Außerdem setzt zu Guttenberg bereits mit diesen einführenden Worten einen Hinweis, dass finanz- sowie haushaltspolitische Gegebenheiten den Handlungsspielraum des Verteidigungsministers in den nächsten Jahren stark beeinflussen könnten.

Im weiteren Verlauf seiner Rede wird zu Guttenberg diese finanz- sowie haushaltspolitischen Bedingungen ausführlicher charakterisieren. Bevor er allerdings auf daraus abzuleitende Entscheidungen zu sprechen kommt, ist der Minister bemüht, ausführlich die Maximen seines Handels herauszustellen. Ihm gehe es darum, ,,[d]en offenen Diskurs [...] [zu] suchen“ (BMVg 2010a, S. 1) und sich ,,bei der Beschreibung der auch bitteren Wirklichkeit vom Prinzip ,Klarheit und Wahrheit" leiten zu lassen“ (ebd., S. 5). Denn er glaube, dass ,ein politischer Stil, der sich [...] im verdrucksten Darstellen äußern würde, am Ende [...] der gesamten Bundeswehr, aber auch unserer Gesellschaft schlichtweg um die Ohren fliegen würde“ (ebd., S. 32).

Jenseits dieser ab Redebeginn durchweg suggerierten Ehrlichkeit und Authentizität seiner Person stellt zu Guttenberg außerdem zu Anfang seines Auftritts Bezüge zu den historischen Gründungsnarrativen der Bundeswehr sowie der Wehrpflicht her, die er am Schluss erneut aufrufen wird. Er betont den Wert der mit den (Leit-)Metaphern Staatsbürger in Uniform sowie Innere Führung verbundenen Errungenschaften und weist auf die erfolgreiche Konzeption der Bundeswehr als demokratische Parlamentsarmee hin (vgl. ebd., S. 8-10, 33). Nach einer Aktualisierung der Gründungsnarrative rückt er diese aber schnell diskursiv wieder in den Hintergrund, da er sie - auch für eine ,Armee im Einsatz“ - für ,,zeitgemäß und notwendig wie ehedem“ (beide Zitate: ebd., S. 8) ansehe. Die Tradition der Bundeswehr sei - so der Tenor - jedenfalls auch in der heutigen Zeit nicht gefährdet - ein wichtiges Signal im Hinblick darauf, welche Überlegungen zur Umstrukturierung der Streitkräfte er noch präsentieren wird.

Nach diesen Feststellungen nähert sich zu Guttenberg dem Thema der Finanzlage des Bundes an. Er betont zwar zunächst noch vage, dass er in seiner Rede am Ende ,noch mal ganz konkret auf das kommen [werde], was [...] mit den aktuell vorliegenden Zahlen in der Folge drohen könnte, in einigen Punkten möglicherweise eben auch droht" (ebd., S. 18). Rückblickend lässt diese Ankündigung aber keinen Zweifel daran, dass hiermit bereits die Aussetzung der Wehrpflicht als zentrales Objekt seines sich entwickelnden Narrativs gemeint ist und er frühzeitig beginnt, eine finanz- bzw. haushaltspolitische Begründung zur Legitimation dieses Vorhabens zu entfalten. So stehe bereits fest, dass im Kontext der damaligen Finanzkrise und ,[v]or dem Hintergrund des absehbaren finanzpolitischen Canossagangs der Bundesregierung [...] nicht nur Rüstungsprojekte auf den Prüfstand“ kommen werden, sondern mit „Einschnitten [...] bei Strukturen und Umfangszahlen“ (beide Zitate: ebd., S. 17) der Bundeswehr zu rechnen sei. Es sei unstrittig, „dass auch der Verteidigungsetat einen Beitrag zur Konsolidierung des Bundeshaushaltes leisten muss“ (beide Zitate: ebd., S. 18). Dies gelte schon deshalb, weil die ,verfassungsrechtlich mittlerweile verankerte Schuldenbremse“ (ebd.) sowie die Verantwortung gegenüber den 
künftigen Generationen ,angesichts des exorbitanten Schuldenbergs“ (ebd., S. 19) Einsparungen gebieten würden (vgl. ebd.).

Um deshalb etwaige Einsparmöglichkeiten zuverlässig festzustellen, habe er innerhalb des Ministeriums seine Staatssekretäre mit eigenen Untersuchungen beauftragt, wobei er diese Ergebnisse in einer noch anstehenden Kabinettsklausur - die später als Sparklausur ${ }^{9}$ bekannt werden sollte - präsentieren wolle (vgl. ebd., S. 20). Auch die laufende Arbeit der neuen Strukturkommission ${ }^{10}$ beschäftige sich mit ,Vorschlägen zur Ausrichtung [...] der Bundeswehr“ (ebd., S. 22), sodass zu Guttenberg mit diesen Schilderungen nicht nur den eigenen Mitarbeitern im Bundesverteidigungsministerium, sondern auch den Akteuren der Bundesregierung sowie jenen der externen Strukturkommission eine wichtige Rolle in seiner Rede zuschreibt. Gemeinsam werden sie von ihm als Helfer in seine Erzählstruktur integriert und - im Falle der Kollegen in Ministerium und Regierung - als Empfänger seiner narrativen Botschaft adressiert. Zusammen können sie außerdem als autoritätsargumentative Instanzen verstanden werden, die den Verteidigungsminister ab dem Zeitpunkt ihrer Nennung mit einer starken narrativen Macht ausstatten, da er die zugewiesenen Akteurskonstellationen fortan als Referenzen bei der Präsentation seiner Pläne nutzen wird. Darüber hinaus lassen sich die Begriffe finanzpolitischer Canossagang, Konsolidierung, Schuldenbremse und Schuldenberg als wichtige (Leit-)Metaphern bezeichnen, die das von zu Guttenberg genutzte finanz- bzw. haushaltspolitische Narrativmuster näher charakterisieren.

Dieses wird im weiteren Verlauf seiner Rede noch komplettiert durch Andeutungen, die sich kaum als spontane, erst am Tag seiner Grundsatzrede angestellte Überlegungen für eine Wehrpflichtaussetzung auffassen lassen. Denn unter Verwendung des Perfekt-Tempus hebt zu Guttenberg - narrativ-rhetorisch geschickt hervor, dass die bundesdeutsche Wehrpflicht, in der Vergangenheit ganz entscheidend zur Einbindung der Bundeswehr in die Gesellschaft beigetragen“ (ebd., S. 24) habe. Er erwarte auch, dass dies ,mit dem auf sechs Monate ehrgeizig verkürzten Wehrdienst der Zukunft so bleibt" (ebd., S. 25), um dann allerdings im gleichen Satz seine Prognose selbst wieder zu relativieren, indem er für die Entscheidung zur Wehrdienstverkürzung herausstellt, dass dieser ,die Haushaltsfrage noch nicht zugrunde lag“ (ebd.). Da sowieso ,für jedes Gesetz der Bewährungstest in der Praxis

\footnotetext{
9 Angesichts der im Jahr 2008 weltweit ausbrechenden Finanz- und Staatsschuldenkrise sah sich die damalige deutsche Bundesregierung zum Sparen gezwungen. Um zu erörtern, welche Beiträge in welchen Ministerien zur Haushaltskonsolidierung denkbar sind, traf sich das Bundeskabinett am 6. und 7. Juni 2010 zu einer Sparklausur (vgl. Lohse und Wehner 2011, S. 312; Kulak et al. 2016, S. 7). Zu Guttenberg nutzte diese Zusammenkunft - wie Lohse und Wehner (2011, S. 313) beschreiben - ,für den ganz großen Aufschlag mitten in der Regierung. Er tritt mit einem Ansatz auf, der geradezu erpresserisch genannt werden kann. Wenn er denn sparen solle wie verlangt, nämlich in einer Größenordnung von 40.000 Zeit- und Berufssoldaten, dann sei die zwingende Folge die Abschaffung der Wehrpflicht, erklärt er kategorisch." Außerdem stellte er in Aussicht, im Rahmen der Strukturveränderungen in der Bundeswehr unter anderem durch eine Wehrdienstaussetzung bis zu 8,3 Mrd. $€$ im Verteidigungsetat einsparen zu können (vgl. Meyer 2010, S. 12).

10 Gemeint ist die von Karl-Theodor zu Guttenberg im April 2010 eingesetzte Strukturkommission der Bundeswehr unter der Leitung des damaligen Vorstandsvorsitzenden der Bundesagentur für Arbeit, FrankJürgen Weise, die bereits ein halbes Jahr später - im Oktober 2010 - ihren vollständigen Bericht an den Minister übergab (vgl. Meyer 2010, S. I, 2; Strukturkommission der Bundeswehr 2010).
} 
erst noch erfolgen muss“, sei in der aktuellen Situation zu bedenken, dass „diese Diskussion [...] nochmal an Schubkraft gewinnen wird, weil plötzlich einige das sehr eng verknüpfen mit der Haushaltsfrage“" (beide Zitate: ebd.). Dabei ist es zuvorderst zu Guttenberg selbst, der diese Verknüpfung vornimmt (vgl. auch Lohse und Wehner 2011, S. 303). Zugleich gelingt es ihm, sich mit den Formulierungen ein (strategisches) Entscheidungsfenster offenzuhalten.

$\mathrm{Zu}$ diesen Ausführungen passt, dass der ehemalige Verteidigungsminister verspricht, den Zuhörern im Laufe seiner Rede ,,noch [...] einen etwas tieferen Einblick“" (BMVg 2010a, S. 28) in die derzeitige Haushaltssituation des Bundes geben zu wollen. Hierbei beschreibt er ,eine von einigen sicher nicht gerne gehörte Paradigmenumkehr“ (ebd., S. 29). Statt einer Bundeswehr nach dem Anspruch Cost to Design werde diese in Zukunft aufgrund der Finanzlage vielmehr ,völlig illusionsfrei durch die Realität des ,Design to Cost' bestimmt werden“ (ebd.). Dem sei sich die ,ganze Bundesregierung, zumal die Spitze“ (ebd.) bewusst, sodass die Regierungskollegen innerhalb der Thematik erneut in einer zentralen Rolle als argumentationsunterstützende Helfer sowie adressierte Empfänger seiner Pläne benannt werden.

Der einprägsamen (Leit-)Metapher Design to Cost sowie dem Schlüsselkonzept Realität stellt zu Guttenberg des Weiteren die den finanz- und haushaltspolitischen Plot verstärkende Überlegung zur Seite, dass strukturelle Veränderungen innerhalb der Bundeswehr - unter anderem zur Senkung der Personalausgaben - unausweichlich seien (vgl. ebd.). Daher werde ,[m]it den jetzt bekannten Zahlen“ unaufhaltsam eine Debatte in Gang kommen, innerhalb welcher nicht zu übersehen sei, „dass in den Streitkräften verbreitet durchaus vor der Disposition von Fähigkeiten das Aussetzen der Wehrpflicht als kurzsichtig wirkende Kompensation auch gesehen wird“" (beide Zitate: ebd., S. 30). Die in dieser Passage kollektiv angesprochenen Bundeswehrverantwortlichen erhalten somit ebenfalls eine wichtige Rollenzuschreibung und werden als weitere Akteure legitimatorisch ins Feld geführt, um die präsentierten Pläne zu stärken. Zwar sind die Bundeswehrangehörigen in Bezug auf die geplanten Reformen damit zunächst als primäre Empfänger der Guttenberg-Narration zu sehen, jedoch werden sie durch den Minister rhetorisch geschickt auch als seine Pläne unterstützende Helfer fokussiert. Außerdem schafft es zu Guttenberg, den Eindruck entstehen zu lassen, als politisch Verantwortlicher mit seinem Vorhaben im Sinne der Streitkräfte zu handeln und sich an ihren Vorschlägen zu orientieren, was ihm zusätzliche narrative Macht verleiht. Denn er arbeite - so möchte er betont wissen - immerzu ,im Sinne der Bundeswehr, der Bundeswehr der Zukunft [...] und nicht aus Eigeninteresse" (ebd., S. 31). ${ }^{11}$

Bereits an dieser Stelle sei jedoch darauf hingewiesen, dass der finanz- bzw. haushaltspolitische Plot nicht bis zum Schluss der Entscheidung zur Wehrpflichtaus-

\footnotetext{
${ }^{11}$ Denn die von ihm in der Grundsatzrede erwähnten Themen seien laut zu Guttenberg „Punkte, da gibt es kein Eigeninteresse mehr. Da kann man nur dramatisch scheitern, wenn man glaubt, da plötzlich politisches Eigeninteresse auf die Waagschale legen zu können“" (BMVg 2010a, S. 31). Obwohl ihm bei seinen Plänen - gerade zur Wehrpflichtaussetzung - durchaus ein gewisses politisches Eigeninteresse zur Selbstprofilierung unterstellt werden darf (vgl. auch Harnisch und Weiß 2014, S. 235), ist doch auffällig, dass zu Guttenberg selbst in seinen Reden dieses immer wieder zurückzuweisen versucht (vgl. auch Bundespresseamt 2010, S. 102, 104).
} 
setzung die dominante Erklärungsvariable bleibt, sondern verstärkt im Sommer 2010 durch einen globalsicherheits- und außenpolitischen Plot abgelöst wird (siehe Abschn. 3.2.3). Die Auswertung der untersuchten Reden zeigt, dass zu Guttenbergs Bundestagsrede am 11. Juni 2010 als vorläufig letzter Beitrag zu lesen ist, der prioritär eine finanzielle Begründung zur Aussetzung des Wehrdienstes enthält (vgl. Deutscher Bundestag 2010c, S. 4815-4816). Ab der zweiten Hälfte des Jahres 2010 wird es dem Minister stattdessen wichtiger hervorzuheben, dass es ,[e]ine Sicherheitspolitik nach Kassenlage [...] nicht geben“ (BMVg 2010b, S. 6) solle. Dies ist im Kontext nach der Grundsatzrede thematisch beginnender politisch-polyphoner Diskussionen einerseits als Reaktion auf Vorwürfe aus den eigenen Reihen der Union zu interpretieren, er richte die Veränderungen der Bundeswehr hauptsächlich nach haushaltspolitischen Gegebenheiten aus (vgl. z. B. Graça Peters 2010; Lohse und Wehner 2011, S. 305). ${ }^{12}$ Andererseits kann eine damalige öffentlichkeitswirksame Intervention von Bundeskanzlerin Angela Merkel (CDU) als wichtige beeinflussende Variable für die Veränderung seines prioritären narrativen Plots gedeutet werden. ${ }^{13}$ Denn maßgeblich durch ihre Vorgaben musste der Verteidigungsminister im Herbst 2010 ,,seine Argumentation vom Kopf auf die Füße stellen und sich eine sicherheitspolitische Begründung für seine Reformpläne ausdenken“ (Lohse und Wehner 2011, S. 315-316). Dabei wurden allerdings sowohl das finanzpolitische als auch das später prioritäre sicherheitspolitische Narrativmuster durch zu Guttenberg seit Beginn der Wehrpflicht-Kontroverse durch eine bundeswehrstrukturell-militärstrategische Argumentation flankiert, wie im Folgenden gezeigt wird.

\footnotetext{
${ }^{12}$ So sagte beispielsweise im Juni 2010 der damalige verteidigungspolitische Sprecher der CDU/CSUBundestagsfraktion, Ernst-Reinhard Beck (CDU), hinsichtlich der von zu Guttenberg zuvor dargelegten finanziellen Begründungen zur Aussetzung des Wehrdienstes, dass er es für wenig sinnvoll halte, „[g]rundsätzliche Pfeiler in Deutschlands Sicherheitsarchitektur nur nach Kassenlage zu hinterfragen“ (Graça Peters 2010). Deutliche Kritik äußerte im selben Monat auch der zu dieser Zeit amtierende CSUParteivorsitzende Horst Seehofer, der die Pläne zur Wehrpflichtaussetzung allerdings eher aus parteipolitischen Gründen ablehnte (vgl. z. B. Süddeutsche Zeitung Online 2010b; Kulak et al. 2016, S. 7). Diese beiden Stimmen stehen dabei stellvertretend für zahlreiche auch in der Folgezeit identifizierbaren Widerstandssignale aus dem politischen Umfeld des Verteidigungsministers, in welchem sich unter anderem die damalige Ministerpräsidentin Thüringens, Christine Lieberknecht (CDU), der frühere Ministerpräsident des Saarlandes, Peter Müller (CDU), oder Bayerns Innenminister Joachim Hermann (CSU) zunächst gegen die von zu Guttenberg präsentierten Ideen stellten (vgl. Spiegel Online 2010b; Kulak et al. 2016, S. 8).

13 So habe Merkel bei einem Unionsfrühstück vor der Sparklausur des Kabinetts im Juni 2010 - bei dem zu Guttenberg allerdings nicht anwesend gewesen sein soll - gesagt, ,,was nicht geht: die Wehrpflicht nur aus finanziellen Gründen abzuschaffen“ (Lohse und Wehner 2011, S. 314). Auch in der Öffentlichkeit zeigte sich die eigentlich ,überzeugte Anhängerin der Wehrpflicht“ hinsichtlich der Veränderungen innerhalb der Bundeswehr zwar offen für die Überlegungen zur Aussetzung des Wehrdienstes, betonte aber, dieser Schritt dürfe ,nicht ausschließlich haushaltspolitisch begründet werden“ (beide Zitate: Süddeutsche Zeitung Online 2010a; vgl. auch Kulak et al. 2016, S. 8-9). Stattdessen müsse es „ein in sich schlüssiges, tragfähiges sicherheitspolitisches Konzept sein, mit dem die Bundeswehr ihren Auftrag erfüllen kann“, weshalb es bei der Wehrpflichtaussetzung ,nicht primär um eine Haushaltsfrage gehen [kann], sondern um eine grundlegende verteidigungs- und gesellschaftspolitische Weichenstellung" (beide Zitate: Spiegel Online 2010a).
} 


\subsubsection{Narratives Muster II: Bundeswehrstrukturell-militärstrategischer Plot}

Der zu identifizierende bundeswehrstrukturell-militärstrategische Plot zeichnet sich zum einen dadurch aus, dass er durchgängig in allen der im Rahmen dieser Narrativanalyse untersuchten Reden wiederzufinden ist. Zum anderen handelt es sich um eine Begründungsstruktur, die zur Wehrpflichtaussetzung zwar in einem Wirkungszusammenhang legitimationsbeschaffend eingesetzt, als Konstante aber von zu Guttenbergs außerdem benutzt wird, um für andere Bestandteile der damaligen Bundeswehrstrukturreform zu werben.

Schaut man detailliert auf das hier untersuchte Muster, so fällt zunächst vor allem der von zu Guttenberg zur Beschreibung der heutigen Bundeswehr genutzte Schlüsselbegriff Armee im Einsatz auf, welchen er sowohl zu Beginn der Jahre 2010 und 2011 in der parlamentarischen als auch Mitte und Ende 2010 in der bundeswehrinternen sowie parteipolitischen Redearena verwendet (vgl. z. B. BMVg 2010a, S. 8, 2010b, S. 5; Bundespresseamt 2010, S. 103; Deutscher Bundestag 2010a, S. 1316, 2011, S. 10424). Das Schlagwort bildet für den Minister den Ausgangspunkt, um die für die Zukunft der Streitkräfte relevanten strukturellen Anforderungen zu definieren. Unter dem Gesichtspunkt sich verändernder militärischer Anforderungen, bei denen für die Bundeswehr ,,[d]er Einsatz und der Kampf [...] stärker in den Mittelpunkt“" (BMVg 2010a, S. 11-12) rücke, sei dann aber ,neben Einsatzbereitschaft auch eine gewisse [...] Opferbereitschaft aufzubringen“ (ebd., S. 12). Nach dem Hinweis, dass dieser Begriff gewissermaßen zwei Dimensionen habe, müsse ,,[d]ie eine [...] im eher abstrakten Sinne sich an einer derzeit vielleicht stattfindenden Finanzdiskussion messen lassen“ (ebd.). Die Überlegung, die Wehrpflicht auszusetzen, um innerhalb der Bundeswehr - so ein wichtiges Schlüsselwort - einer angemessenen „Einsatzfähigkeit“ (ebd., S. 11; Hervorh. d. Verf.) Rechnung zu tragen, nutzt zu Guttenberg in diesem Zusammenhang als narrativ einprägsames Argument. Denn das vom Einsatz her orientierte Denken der künftigen Bundeswehrstruktur müsse ,,quasi der kreative Vorwegabzug für das Fortbestehen der Streitkräfte sein. Erst dahinter können solitäre Elemente der allgemeinen Sicherheitsvorsorge erhalten werden“ (ebd., S. 30).

Insgesamt prägen ähnliche Argumentationsstrukturen auch die folgenden Reden des Verteidigungsministers innerhalb wie außerhalb des Bundestages bis Februar 2011. Zwar werden in diesen bundeswehrstrukturell-militärstrategische Erzählstrukturen ebenso angeführt, um später eine sicherheitspolitische Argumentation narrativ zu etablieren, trotzdem werden eine Orientierung an den „Erfordernisse[n] des Einsatzes“ und das Ziel „eine[r] verbesserte[n] Leistungsfähigkeit“ (beide Zitate: Deutscher Bundestag 2010d, S. 6105) von ihm als wichtige Maximen des zukünftigen Handelns unabhängig von anderen Plots beibehalten. Um das funktionale Aufgabenspektrum der Bundeswehr zu erfüllen sowie ,flexibler und besser zu werden“ (ebd.), sei außerdem ein verminderter Gesamtumfang der Truppe von Nöten (vgl. ebd.). Denn nur so sei ,die militärische Handlungsfähigkeit Deutschlands sicherzustellen und ein der Stellung Deutschlands angemessenes, akzeptables Fähigkeitsprofil zu bieten“ (BMVg 2010b, S. 7), so zu Guttenbergs Begründungsmuster vor der bayerischen Frauenunion im September 2010. Dieses kann aufgrund des Schlüsselworts militärische Handlungsfähigkeit nahezu als Zusammenfassung sei- 
ner bundeswehrstrukturell-militärstrategischen Argumentation zur Wehrpflichtaussetzung gelesen werden.

Durch die Tatsache, dass bezüglich der Abwägungen zur Verkleinerung der Bundeswehr in der zweiten Hälfte des Jahres 2010 vermehrt Modelle und Berichte zur zukünftigen Struktur der Streitkräfte in den öffentlichen Diskurs eingebracht und in der polyphonen Öffentlichkeit diskutiert werden (vgl. z. B. Meyer 2010, S. 12-14; Schmidt 2010; Harnisch und Weiß 2014, S. 227, 229-230), beginnt in der Folgezeit auch der Minister, diese neuen Konzepte in seinen Reden stärker als Legitimationsinstanzen miteinzubeziehen. Den von zu Guttenberg bei seinen Auftritten namentlich erwähnten Autoren dieser konzeptionellen Pläne ist daher eine gewichtige narrative Rolle als Helfer zuzumessen.

Um sein Wehrpflichtaussetzungsnarrativ argumentativ mithilfe an der Thematik beteiligter Akteure zu stärken, führt zu Guttenberg innerhalb wie außerhalb des Bundestages beispielsweise im September und November 2010 sowie im Februar 2011 die bereits kurz erwähnte ministeriumsinterne Defizitanalyse seiner Mitarbeiter sowie ein ausgearbeitetes Papier des damaligen Generalinspekteurs der Bundeswehr, Volker Wieker, an. Ferner bezieht er sich auf Empfehlungen im Bericht der Strukturkommission der Bundeswehr (vgl. CDU Deutschland 2010a, S. 129, 131; Deutscher Bundestag 2010d, S. 6105, 2011, S. 10424). ${ }^{14}$ Dieser Bericht war am 26. Oktober 2010 veröffentlicht sowie dem Minister übergeben worden und sprach sich für eine neue Bundeswehrstruktur im Allgemeinen sowie für eine Wehrpflichtaussetzung im Besonderen aus (vgl. Meyer 2010, S. 2; Strukturkommission der Bundeswehr 2010, S. 26-29; siehe auch FN 10). Mit der Aussagekraft des Titels „Vom Einsatz her denken. Konzentration, Flexibilität, Effizienz" (Strukturkommission der Bundeswehr 2010, S. 1) kann die Kommissionsanalyse danach von zu Guttenberg als wichtige Referenz in seine bundeswehrstrukturell-militärstrategische Argumentation eingebaut werden (vgl. z. B. Bundespresseamt 2010, S. 113, 116). Umgekehrt stützen die Analyseempfehlungen gleichzeitig auch zu Guttenbergs Narration, wobei der Minister diese später zudem nutzen wird, um die Wehrpflichtaussetzung sicherheitspolitisch zu begründen (siehe Abschn. 3.2.3).

In Bezug auf das bundeswehrstrukturell-militärstrategisch Narrativmuster sei jedoch zunächst noch ergänzt, dass es zu Guttenberg fortlaufend wichtig ist, das Aus des Wehrdienstes in den Kontext einer visionären „Neuausrichtung“ (z. B. Deutscher Bundestag 2010f, S. 8838, 2011, S. 10426) der Bundeswehr zu stellen. Nicht nur eine finanzielle Grundlage bilde den Maßstab der Veränderungen, sondern die seinerseits als weitblickend herausgestellte Anforderung, die anstehende „Reform nicht als Selbstzweck“ (Deutscher Bundestag 2010e, S. 8135) durchzuführen, um die Bundeswehr ,zukunftsfest auf[zu]stellen, damit sie eine Perspektive entwickeln kann“ (Deutscher Bundestag 2011, S. 10425, vgl. auch 2010e, S. 8135). Diese an

\footnotetext{
14 Schon in seiner Grundsatzrede im Mai 2010 hatte der Verteidigungsminister bezüglich der Strukturkommission nachdrücklich den seinerseits zugemessenen, wichtigen Stellenwert des Formats und ihrer damals noch ausstehenden - Ergebnisse betont. Wörtlich sagte er: „Nachdem sich selbst meine Geduld mit Arbeitsgruppen und Kommissionen in messbaren Grenzen hält, weil wir dazu eine gewisse Neigung im politischen Bereich haben, diese einzusetzen und am Ende des Tages sie mit großen Worten wieder zu verabschieden, aber nicht umzusetzen, soll jener Anspruch ein anderer sein und auch in eine entsprechende Umsetzung münden“ (BMVg 2010a, S. 14).
} 
eine Neuausrichtung auf der Grundlage einer Einsatzorientierung gebundene Perspektive - so sind die drei Schlüsselwörter kontextuell zu interpretieren - lasse sich allerdings nur durch ein Ende der Wehrpflicht zuverlässig gewährleisten.

Darüber hinaus sei - wie der Minister seit Mitte des Jahres 2010 anführt - ein verantwortungsvolles „Denken vom Einsatz her [...] etwas, was wir letztlich schon seit 20 Jahren als Realität begreifen müssen [...]. Es ist Realität und es wird Realität bleiben“ (Deutscher Bundestag 2010c, S. 4816). Bei näherer Betrachtung dieser Redepassagen fällt auf, dass es hier wiederum das einprägsame Schlüsselwort Realität ist, welches zu Guttenberg in verschiedenen Rede- und Argumentationskontexten benutzt (vgl. z. B. auch Bundespresseamt 2010, S. 114; CDU Deutschland 2010a, S. 137). Als ein verbindlichkeitsherstellendes Konzept verstärkt es so mit einer enormen narrativen Legitimationskraft die durch den homo narrans sprachlich dargestellten Zusammenhänge. ${ }^{15}$

Überdies zeichnet sich die bundeswehrstrukturell-militärstrategische Argumentation durch ein letztes Merkmal aus, nämlich dass durch den Minister bundeswehrinterne Abläufe beschrieben werden, die eine Wehrpflichtaussetzung als logische Schlussfolgerung nahelegen. Wenn zu Guttenberg schon im Juni 2010 beschreibt, dass bei einer Beibehaltung der Wehrpflicht und gleichzeitigen Personalkürzungen innerhalb der Bundeswehr ,,möglicherweise zu viele der länger dienenden Soldaten durch rein wehrpflichtspezifische Aufgaben [...] gebunden wären" (Deutscher Bundestag 2010c, S. 4816; vgl. auch BMVg 2010b, S. 8-9), verdeutlicht dies von Anfang an die Ausrichtung seiner Argumentation. Denn ,[d]ie damit verbundene enorme Kraftanstrengung würde an die Grenze ihrer Vertretbarkeit, aber auch ihrer Vermittelbarkeit in der Truppe stoßen“ (Deutscher Bundestag 2010c, S. 4816). Wie er in seiner Rede vor dem Bundestag im Februar 2011 betont, sei dies letztlich auch ein entscheidendes Argument gewesen, ,weshalb wir gesagt haben: Wir können künftig den Grundwehrdienst nicht mehr so wie ursprünglich aufrechthalten“ (Deutscher Bundestag 2011, S. 10425). Hierbei ist zu bemerken, dass zu Guttenberg mit seiner Wortwahl - neben einer inhaltlichen Betonung ausbildungsstruktureller Gründe - eine starke Ausrichtung an den Erfordernissen der Truppen heraushebt und so die Bundeswehrsoldaten als legitimationsbeschaffende Helfer sowie an der politischen Entscheidung mitwirkende Akteure in sein Gesamtnarrativ einbezieht. Dazu passt auch, dass der Minister bereits in seiner Dresdner Rede Ende 2010 vor Verantwortlichen der Bundeswehr betont, sich „in der Pflicht für diese Bundeswehr“ (Bundespresseamt 2010, S. 119) zu sehen und mit der „Unterstützung, [...] Loyalität und [...] Gestaltungskraft“" (ebd.) der Truppe die zukünftigen Aufgaben angehen zu wollen.

\footnotetext{
15 Besonders anschaulich wird dies auch beispielsweise in seiner CDU-Parteitagsrede Mitte November 2010, in der zu Guttenberg die Diskussion um die Wehrpflichtaussetzung ganzheitlich damit begründet, dass ,sich die Realitäten nicht erst in den letzten zwei Jahren entwickelt haben. Es handelt sich vielmehr um einen Entwicklungsprozess, der in den letzten 20 Jahren abgelaufen ist. Wir müssen den Realitäten ins Auge blicken. Wir haben möglicherweise vor manchen dieser Realitäten die Augen verschlossen“ (CDU Deutschland 2010a, S. 132).
} 


\subsubsection{Narratives Muster III: Globalsicherheits- und außenpolitischer Plot}

Wie im Verlauf der Analyse bereits angedeutet, kennzeichnet das Wehrpflichtaussetzungnarrativ zu Guttenbergs in Gänze, dass der Minister seine anfänglich genutzte finanz- bzw. haushaltspolitische Argumentation (siehe Abschn. 3.2.1) zum Ende des Jahres 2010 immer stärker revidiert und durch ein plotbildendes globalsicherheitsbzw. außenpolitisches Narrativmuster ersetzt. Als mögliche Anstöße für diese Veränderungen wurden dabei die im Sommer 2010 ansteigende politisch-polyphone Kritik an der Wehrpflichtaussetzung sowie eine Intervention Angela Merkels gedeutet (siehe auch FN 12 und 13). Tatsächlich beginnt zu Guttenberg daraufhin im Herbst des gleichen Jahres, seine anfänglich zur Durchsetzung des Wehrpflichtendes angeführte monetäre durch eine sicherheitsorientierte Argumentation zu ersetzen.

So führt der damalige Bundesminister der Verteidigung erstmals in seiner Rede anlässlich der Fachkonferenz Zukunft Bundeswehr mit Entschiedenheit aus, dass „die Strukturen von Streitkräften stets an die sicherheitspolitischen Herausforderungen der jeweiligen Zeit angepasst werden müssen“ (BMVg 2010b, S. 3). Außerdem sei die heutige globalisierte Welt ,in sicherheitspolitischer Hinsicht nicht mehr mit der Zeit seit der Gründung der Bundeswehr 1955 bis zum Fall des Eisernen Vorhangs vergleichbar" (ebd.), sodass die Bundeswehr entsprechend ausgerichtet werden sollte (vgl. ebd.). Insbesondere bestehe keine ,unmittelbare territoriale Bedrohung Mitteleuropas und damit Deutschlands [...] mehr" (ebd., S. 4), sondern stattdessen würden sich die deutschen Truppen verstärkt asymmetrischen Bedrohungen außerhalb des eigenen Bündnisgebiets ausgesetzt sehen (vgl. ebd., S. 8). Dabei gelte es aber zu bedenken, dass Wehrpflichtige aufgrund der verfassungsmäßigen Bestimmungen des Wehrdienstes ,in diesen Einsatzszenarien nicht unmittelbar einsetzbar" (ebd.) seien (vgl. auch Deutscher Bundestag 2010d, S. 6106). ${ }^{16}$ Im Vergleich zum vorherig explizierten, finanz- bzw. haushaltspolitischen Narrativmuster wird somit schon bei Betrachtung dieser ersten ausgewählten Redeausschnitte deutlich, dass zu Guttenberg nun eine sichtbare Prioritätenverschiebung in seiner Argumentation vornimmt. Statt der (Leit-)Metaphern wie Schuldenbremse oder Schuldenberg prägen jetzt die Schlüsselwörter sicherheitspolitische Herausforderungen und neue Einsatzszenarien das von ihm entwickelte narrative Muster.

Aufgrund der veränderten sicherheitspolitischen Gegebenheiten müssten - so der Verteidigungsminister - nunmehr unmittelbare Veränderungen in der Bundeswehr vollzogen werden. Obschon diese „Notwendigkeit“ (BMVg 2010b, S. 5) existiere, dürfe es aber keine „Sicherheitspolitik nach Kassenlage [...] geben“ (ebd., S. 6) oder ,[d]ie Schutzfunktion der Bundeswehr [...] durch keinen Sparbeschluss der Welt in Frage gestellt werden“" (ebd., S. 7). Wenn zu Guttenberg stattdessen fordert, „sicherheitspolitisch unnötigen Ballast abzuwerfen“ (ebd.), so ist dieser Ballast in

\footnotetext{
16 Ergänzend ist zu vermerken, dass - durch die Gründungsbestimmung der Bundeswehr als Truppe zur eigenen Landes- und Bündnisverteidigung - die in ihr bis Mitte des Jahres 2011 dienenden Wehrpflichtigen tatsächlich alleinig im Rahmen eines festzustellenden Verteidigungsfalls hätten eingesetzt werden können (vgl. Raidel 1998, S. 14, 21-23; Rose 1998, S. 60). Die für die Bundeswehr nach dem Ende des Ost-West-Konflikts wichtiger werdenden internationalen Auslandseinsätze konnten also ohnehin nur mit Berufssoldaten ausgeführt werden, da die Wehrpflichtigen nicht in ausländische Einsatzgebiete geschickt werden durften (vgl. Meyer 2010, S. 25).
} 
der Rückschau als (Leit-)Metapher für die Wehrpflicht zu lesen. Zugleich etabliert sich das Wort Kassenlage als ein Schlüsselkonzept innerhalb seines neu konstruierten Plots, das auch öffentlichkeitswirksam in der (Presse-)Berichterstattung über die Wehrpflichtaussetzung oft zitiert wurde (vgl. z. B. Frankfurter Allgemeine Zeitung Online 2010a).

Um sein plotbildendes globalsicherheits- und außenpolitisches Narrativmuster im Folgenden noch weiter zu stärken, bemüht sich zu Guttenberg um weitere Formulierungen, welche die Bedeutung seiner inzwischen sicherheitspolitischen Erzählweise erkennen lassen. So sei der Wehrdienst in der aktuellen Ausgestaltung ,sicherheitspolitisch nicht begründbar“ (BMVg 2010b, S. 8; vgl. auch Bundespresseamt 2010, S. 101). Daher lege die heutige sicherheitspolitische Lage ,ein Aussetzen der Allgemeinen Wehrpflicht in ihrer bisherigen Form nahe. Dem Beitrag zur Haushaltskonsolidierung kommt hierbei lediglich eine Katalysator-Funktion zu“ (BMVg 2010b, S. 9).

Dass der Verteidigungsminister mit diesen Aussagen innerhalb kürzester Zeit seine anfänglich genutzten finanzpolitischen Argumente und seine darin konstruierten Kausalmuster wieder einzufangen versucht, scheint nun offensichtlich. Dass er damit aber gleichsam innerhalb von nur fünf Monaten auch im Hinblick auf seine sicherheitspolitischen Einschätzungen eine narrative 180-Grad-Drehung vollzieht, kann zunächst nicht erkannt werden. Dies wird erst bei einer vergleichenden Betrachtung der obigen Äußerungen mit einer im Mai 2010 stattgefunden Debatte im Deutschen Bundestag deutlich, bei welcher der Minister bemüht war, die zu dieser Zeit geplante Wehrdienstverkürzung auf sechs Monate noch sicherheitspolitisch zu legitimieren (vgl. Deutscher Bundestag 2010b, S. 4194-4201). ${ }^{17}$

Die getroffene Feststellung, dass zur Begründung der Wehrpflichtaussetzung bis zum Ende des Jahres 2010 verstärkt der globalsicherheits- bzw. außenpolitische Plot überwiegt, lässt sich ferner anhand der weiteren, hier untersuchten Reden aufzeigen. Jenseits der in die Wehrpflicht-Debatte eingebrachten polyphonen Signale der unionsinternen Kritiker und durch Bundeskanzlerin Merkel werden dabei abermals die erwähnten Modelle zur zukünftigen Struktur der Bundeswehr durch Generalinspekteur Wieker sowie der Bericht der Strukturkommission der Bundeswehr als Legitimationsinstanzen wichtig, denen zu Guttenberg zentrale helfende Rollen zumisst. Wie der Verteidigungsminister nämlich am 15. September 2010 im Bundestag erklärt,

\footnotetext{
17 Diese Tatsache lässt sich exemplarisch durch eine Stellungnahme zu Guttenbergs nach einer Frage von Agnes Malczak (Bündnis 90/Die Grünen) bei der am 19. Mai 2010 durchgeführten Befragung der Bundesregierung im Bundestag belegen. Denn diese hatte zu Guttenberg vorgeworfen, ,in den Monaten, in denen über diese Reform [der Wehrdienstverkürzung auf sechs Monate, Anm. d. Verf.] intensiv diskutiert wurde, eine fundierte sicherheitspolitische Begründung“ (Deutscher Bundestag 2010b, S. 4198) zu vermissen. Auf ihre Nachfrage, ob der Verteidigungsminister für die Wehrdienstverkürzung eine solche nun geben könne, antwortete er: „Zu einer sicherheitspolitischen Begründung zählt natürlich weiterhin die Funktionsfähigkeit der Armee, auch im Hinblick auf die Betriebs- und Schutzfunktionen im Inland. Das ist Grundvoraussetzung dafür, dass Einsätze auch im Ausland funktionieren. Um diese Funktionsfähigkeit zu gewährleisten, sind wir weiterhin darauf angewiesen, zielgerichtet auf Wehrpflichtige zurückzugreifen. Das passt sehr wohl zum großen Bogen der sicherheitspolitischen Herausforderungen und damit zur Begründung“ (ebd.). Dass zu Guttenberg seine Ansichten in dieser Frage nur wenige Monate später - ohne grundsätzlich veränderte sicherheits- oder außenpolitische Gegebenheiten innerhalb dieser Zeit - gänzlich ändert, kann daher erstaunen, bestätigt aber den vollzogenen Wandel in seinem Narrativ.
} 
habe seine geplante Reform ,zunächst einmal eine sicherheitspolitische Analyse zur Grundlage“, welche „die sicherheitspolitischen Herausforderungen der Gegenwart und der Zukunft“ (beide Zitate: Deutscher Bundestag 2010d, S. 6105) berücksichtige. Letztere habe „der Generalinspekteur der Bundeswehr zu Papier gebracht“, wobei diese Untersuchung ,die Grundlage für die kommenden Schritte darstellen [...] soll“ (ebd.). Hier dürfe es dann aber „,nicht allein um die Frage gehen, was wir uns noch leisten können. Die sicherheitspolitische Grundlage ist das Maßgebliche“" (ebd., S. 6107).

Komplettiert wird diese, auf im Laufe der Zeit einbezogene Helfer als Autoritätsinstanzen basierende sicherheitspolitische Begründungsstruktur von zu Guttenberg durch mehrmalige öffentlichkeitswirksame Erwähnung der Empfehlungen im Bericht der Strukturkommission der Bundeswehr (vgl. z. B. CDU Deutschland 2010a, S. 128; Bundespresseamt 2010, S. 105; siehe auch Abschn. 3.2.2). Dieser sprach sich bei der anstehenden Streitkräftereform dafür aus, „[d]ie Musterung und Einberufung der Wehrpflichtigen zum Grundwehrdienst auszusetzen“ und stattdessen „[e]inen freiwilligen, bis zu 23-monatigen Dienst einzuführen“ (beide Zitate: Strukturkommission der Bundeswehr 2010, S. 28). Als Grund für diese Empfehlung vermerkte die Kommission, dass ,[d]urch den Wegfall einer massiven, unmittelbaren militärischen Bedrohung die Wehrpflicht in der heutigen Form sicherheitspolitisch nicht mehr gerechtfertigt werden“ (ebd.) könne. Damit bot diese Passage für den Verteidigungsminister einen weiteren narrativen Anknüpfungspunkt, um die Wehrpflichtaussetzung mit einer fehlenden sicherheitspolitischen Notwendigkeit des Wehrdienstes zu plausibilisieren. ${ }^{18}$ Außerdem ermöglichte ihm die Argumentation um das omnipräsente Schlüsselkonzept Sicherheit nicht zuletzt eine wirksame eigene Profilierung, da er schließlich bei einer Diskussion im Bundestag Ende 2010 „hocherfreulich“ feststellen konnte, dass man nun über eine zukünftige Bundeswehrstruktur Einigkeit erzielt habe, ,die sich über die sicherheitspolitischen Herausforderungen und Erfordernisse definiert" (beide Zitate: Deutscher Bundestag 2010e, S. 8134).

Ein zu ergänzender Aspekt, der sein globalsicherheits- bzw. außenpolitisches Narrativmuster zusätzlich festigt, ist die durch den Minister erstmals explizit auf dem CDU-Parteitag sowie bei der Dresdner Bundeswehrtagung im November 2010 thematisierte Feststellung, dass durch seine Pläne ,[w]eder die verfassungsrechtliche noch die einfachgesetzliche Grundlage der Wehrpflicht [...] gänzlich abgeschafft" (Bundespresseamt 2010, S. 133) werde. Es gehe nicht darum, die allgemeine Wehrpflicht ,komplett [zu] beerdigen und aus dem Grundgesetz [zu] streichen“ (CDU Deutschland 2010a, S. 137). Diese müsse vielmehr dort verankert bleiben, denn „[w]er weiß, wie sich die Welt in 10, 15 oder 20 Jahren entwickelt?“ (ebd.; vgl.

\footnotetext{
18 Anzumerken sei, dass diese Begründung auf einer historisch-diskursiven Argumentationslinie zu verorten ist. Denn schon der ehemalige Bundespräsident Roman Herzog (CDU) hatte im Jahr 1995 die sicherheitspolitische Notwendigkeit als zwingende Beurteilungsgrundlage für die bundesdeutsche Wehrpflicht herausgestellt. Damals betonte er: „Die Wehrpflicht ist ein so tiefer Einschnitt in die individuelle Freiheit des Bürgers, dass ihn der demokratische Rechtsstaat nur fordern darf, wenn es die äußere Sicherheit des Staates wirklich gebietet. Sie ist also kein allgemeingültiges ewiges Prinzip [...]. Ihre Beibehaltung, Aussetzung oder Abschaffung [...] müssen sicherheitspolitisch begründet werden können“ (zit n. Rose 1998, S. 59).
} 
auch Deutscher Bundestag 2011, S. 10425). Dass zu Guttenberg die Wehrpflicht nur aussetzen und nicht abschaffen will, folgt somit also zum einen seinem kausalen Erzählmuster, das vordergründig sicherheitspolitische Erwägungen narrativ priorisiert, indem weitsichtig auf eine kaum kalkulierbare Zukunft der außenpolitischen Lage vorausgeblickt wird. Zum anderen kann angenommen werden, dass die herausgestellte Bedeutungsdifferenzierung eine Reaktion auf sein polyphones politisches Umfeld darstellt und widerständige Bundeswehrverantwortliche sowie Parteimitglieder besänftigen sollte. ${ }^{19}$

Unter diesem letzten Gesichtspunkt ist gleichermaßen zu interpretieren, weshalb zu Guttenberg auf dem CDU-Parteitag betont, die Wehrpflicht nicht aus ,,ideologischen Gründen“ (CDU Deutschland 2010a, S. 137) aussetzen zu wollen. Er selbst gehöre zwar immer noch ,zu den wohl glühendsten Verfechtern der Wehrpflicht“ (ebd., S. 131), es gelte nun aber - hier findet sich einmal mehr die Verwendung des Schlüsselworts Realität - ,in der Sicherheitspolitik und mit Blick auf die Bundeswehr den Realitäten ins Auge zu blicken“ (ebd., S. 137). Dass der nach der Rede zu Guttenbergs von den Delegierten angenommene CDU-Parteitagsbeschluss zur Zukunft der Bundeswehr (vgl. ebd., S. 162, 285) dann primär sicherheitspolitische Begründungen für die Aussetzung der Wehrpflicht enthält, gleichzeitig haushaltspolitische Erwägungen revidiert (vgl. CDU Deutschland 2010b, S. 1-3) und damit dem gewandelten Wehrpflichtaussetzungsnarrativ des Verteidigungsministers in Gänze folgt, kann nicht verwundern. ${ }^{20}$

Die dargelegte Paradigmenumkehr passt zudem aber noch zu einer weiteren Auffälligkeit, die bislang kaum erkannt wurde: Ging es zu Guttenberg nämlich bei seinen finanziellen Argumentationen bis Mitte des Jahres 2010 vornehmlich darum, die haushaltspolitische Notwendigkeit zur Kürzung der Bundeswehr-Personalausgaben zu betonen und eine daraus resultierende Unvereinbarkeit des Wehrdienstes mit einer verkleinerten Beschäftigungszahl zu begründen, so scheinen diese finanziellen Einsparungen beim Personal durch den Wandel hin zu einem sicherheitspolitischen Plot zum Ende des selben Jahres allenfalls noch randständig relevant.

Besonders deutlich wird dieser Aspekt bei der Rede zu Guttenbergs im Rahmen der zweiten Bundestagsberatung des Bundeshaushaltsplans für das Haushaltsjahr 2011 am 24. November 2010. Nach einem dortigen Zwischenruf, der den Verteidigungsminister damit konfrontiert, ob er den in der Kabinettsklausur der Bundesregie-

\footnotetext{
${ }^{19}$ Denn dass die Wehrpflicht am Ende tatsächlich lediglich ausgesetzt und nicht abgeschafft wurde, hat das - für einige Unionspolitiker damals sicherlich kompromissfähige - Resultat zur Folge, dass sie jederzeit mit der Verabschiedung eines einfachen Gesetzes wieder eingeführt werden könnte (vgl. Meyer 2010, S. 19).

${ }^{20}$ So heißt es in dem Beschluss: „Mit dem Ende des Kalten Krieges hat sich die sicherheitspolitische Lage Deutschlands grundlegend verändert. Seitdem ist unser Land ausschließlich von Freunden und Partnern umgeben [...]. Die größten Bedrohungen gehen heute von Terroristen, diktatorischen Regimen sowie instabilen und zerfallenen Staaten aus [...]. Auf diese Herausforderungen muss die Bundeswehr stärker als bisher ausgerichtet werden [...]. Dies zeigt auch: Bei allen Transformationsüberlegungen geht es nicht darum, die Bundeswehr billiger, sondern für die aktuellen und zukünftigen Herausforderungen besser aufzustellen. Die Wehrreform darf nicht von der Haushaltslage bestimmt sein [...]. Die Präsidien von CDU und CSU teilen die Sicherheitsanalyse des Bundesministers der Verteidigung [...]. Schlussfolgerung aus der Analyse ist, dass eine sicherheitspolitische Notwendigkeit für die allgemeine Wehrpflicht nicht mehr gegeben ist“ (CDU Deutschland 2010b, S. 1-2).
} 
rung im Juni 2010 zur Haushaltskonsolidierung genannten Sparbeitrag des Verteidigungshaushaltes von 8,3 Mrd. $€$ mit seinen ausgearbeiteten Reformplänen einhalten könne (vgl. Deutscher Bundestag 2010e, S. 8135; siehe auch FN 9), antwortete der Minister, dass dieses Ziel nur „schwierig“ (ebd.) zu beachten sei. Denn dies sei ein Zahlenrahmen gewesen, ,den man mit etwa 150.000 Soldaten erreicht hätte“ (ebd.). Wenn nun aber eine Zahl von 180.000-185.000 Soldaten im Raum stehe, dann ,wird man ihr gerecht werden müssen“ (ebd.). ${ }^{21}$ Dass zu Guttenberg mit diesen komplexen Formulierungen einen an die veränderten Soldatenzahlen orientierten, finanziellen Mehraufwand meint und erzählerisch behutsam von seinen anfänglichen Sparplänen abrückt, lässt sich dabei erst bei mehrmaliger Betrachtung der Redepassage verstehen. Zugleich verbindet er die Abkehr von seinen ursprünglichen Versprechungen jedoch mit seinem neuen sicherheitspolitischen Narrativmuster. Denn er wolle eine Bundeswehr ausgestalten, die ,ein [...] kluges Aufgabenspektrum vorhalten [...] kann“ (ebd., S. 8136).

Zieht man jenseits der hier untersuchten Reden zur Überprüfung dieser Interpretation die Haushaltspläne des Bundesministeriums der Verteidigung nach dem Jahr 2010 heran, zeigt eine Auswertung dieser Soll-Aufstellungen ${ }^{22}$, dass sich trotz Aussetzung der Wehrpflicht tatsächlich in den Folgejahren kaum Einsparungen im Verteidigungsetat eingestellt haben. Stattdessen erhöhten sich - mit Ausnahme des Jahres 2014 - die eingeplanten Gesamtausgaben im Verteidigungshaushalt seit 2010 stetig, obwohl zu Guttenberg im Bundeskabinett Mitte 2010 angekündigt hatte, die Einsparungen von 8,3 Mrd. $€$ bis 2014 erreichen zu wollen (vgl. Meyer 2010, S. 12). ${ }^{23}$ Analysiert man nur die kalkulierte Entwicklung der Personalausgaben, ergibt sich zwar ein differenzierteres Bild, doch auch dieses lässt kein Erreichen des ursprünglich zugesagten Sparpotenzials erkennen. ${ }^{24}$ Obwohl berücksichtigt werden muss, dass Karl-Theodor zu Guttenberg durch seinen Rücktritt als Bundesminister

\footnotetext{
21 Ähnlich hatte sich zu Guttenberg auch schon in seiner Rede zwei Tage zuvor bei der Dresdner Bundeswehrtagung geäußert: Zwar sei ein Streitkräfteumfang zwischen 180.000 und 185.000 Soldaten geeignet, um den ,absehbaren sicherheitspolitischen Anforderungen in angemessener Weise Rechnung [zu] tragen“ (Bundespresseamt 2010, S. 109). Allerdings gelte dies nur ,unter der Voraussetzung hinreichender Finanzierung" (ebd.).

22 Etwaige Nachtragshaushalte konnten im Einzelnen nicht berücksichtigt werden.

23 Betrugen die Gesamtausgaben des Bundesministeriums der Verteidigung im Jahr 2010 noch rund 31,11 Mrd. $€$, so lagen diese im Jahr 2011 schon bei 31,55 und 2012 bei 31,87 Mrd. €. Nachdem im Jahr 2013 die Ausgaben sogar um circa 1,4 Mrd. auf insgesamt rund 33,26 Mrd. $€$ gestiegen waren, ließ sich für 2014 ein kleiner Rückgang um 0,82 Mrd. $€$ auf 32,44Mrd. $€$ erkennen. Bis zum Jahr 2018 sind die Verteidigungsausgaben dann aber ohne Ausnahme bis auf 38,52 Mrd. $€$ angestiegen, wobei für das Jahr 2019 sogar Gesamtausgaben von 43,23 Mrd. € eingeplant waren (vgl. BMF 2010, S. 118, 2011, S. 115, 2012, S. 115, 2013, S. 3, 2014, S. 3, 2015, S. 3, 2016, S. 4, 2017, S. 4, 2018, S. 5, 2019, S. 5).

24 Beliefen sich die Personalausgaben des Verteidigungsministeriums im Jahr 2010 auf circa 16,36 Mrd. €, so lagen sie 2011 schon bei rund 16,53 Mrd. $€$. Eine Minderung dieses Betrags weisen danach die Personalausgaben des Jahres 2012 mit 14,92 Mrd. $€$ auf, ehe sie im Jahr 2013 wieder auf 15,77 Mrd. $€$ anstiegen. 2014 erfolgte kurzzeitig eine erneute Abnahme auf 15,69 Mrd. €. Bis zum Jahr 2018 sind dann mit einem Anstieg auf rund 17,9 Mrd. $€$ jedoch keine zwischenzeitlichen Rückgänge in den Personalausgaben des Verteidigungsetats mehr zu verzeichnen. Zudem war für das Jahr 2019 eine weitere Erhöhung auf circa 18,76 Mrd. $€$ vorgesehen (vgl. BMF 2010, S. 118, 2011, S. 115, 2012, S. 115, 2013, S. 3, 2014, S. 3, 2015, S. 3, 2016, S. 4, 2017, S. 4, 2018, S. 5, 2019, S. 5).
} 
der Verteidigung Anfang März $2011^{25}$ die Bundeswehrreform wie auch die Wehrpflichtaussetzung selbst nicht mehr politisch ausgestalten konnte, so erstaunt rückblickend doch, dass letztere ohne weitere nennenswerte (finanzielle) Diskussionen noch am Ende des selben Monats im Bundestag verabschiedet und wie geplant zum 01. Juli 2011 umgesetzt wurde (vgl. Kulak et al. 2016, S. 16-17). ${ }^{26}$ Schließlich stand zu diesen Zeitpunkten - trotz des erstmaligen Greifens der Schuldenbremse und des Drucks für die Bundesregierung, nach der 2008 ausbrechenden Finanzkrise Einsparungen vornehmen zu müssen - für das Jahr 2011 eine Erhöhung der Verteidigungsausgaben im Allgemeinen sowie der Personalausgaben innerhalb des Verteidigungsetats im Besonderen längst fest, da der entsprechende Haushaltsplan schon am 26. November 2010 den Bundestag passiert hatte (vgl. Frankfurter Allgemeine Zeitung Online 2010b).

Im Kontext der hier durchgeführten Narrativanalyse kann die dennoch erfolgte Aussetzung des Wehrdienstes somit auf eine insgesamt starke Überzeugungskraft der Gesamterzählung zu Guttenbergs zurückgeführt werden. Dabei wurde sein Wehrpflichtaussetzungsnarrativ im Zeitverlauf seiner Redeauftritte inhaltlich immer stärker sicherheitspolitisch flankiert, bis letztlich der die Debatte initiierende finanzpolitische Narrativbestandteil nach September 2010 derart stark zu bröckeln begann, dass dieser für die endgültige Entscheidung kaum mehr eine Rolle spielte.

\subsubsection{Narratives Muster IV: Verfassungsrechtlich-gesellschaftspolitischer Plot}

Wie eine Durchsicht aller untersuchten Reden offenbart, handelt es sich bei der globalsicherheits- und außenpolitischen Argumentation allerdings nicht um das einzige Narrativmuster, welches zu Guttenberg zur Legitimierung der Wehrpflichtaussetzung in seinen späteren Reden entwickelt. Obwohl dieses in seinen Auftritten ab Mitte des Jahres 2010 in der Häufigkeit der Akzentuierung überwiegt, belässt er es nicht bei einer monokausalen Begründung für sein Vorhaben. Stattdessen entfaltet er bis Februar 2011 einen weiteren Plot, der Aspekte einer verfassungsrechtlichen wie gesellschaftspolitischen Begründung einbezieht.

So deutet der Verteidigungsminister die Problematik einer seit den letzten Jahren verstärkt bestehenden Wehrungerechtigkeit als gesellschaftspolitisches Argumentationsmuster zur Aussetzung des Wehrdienstes erstmals nachdrücklicher bei seiner Rede im Bundestag Mitte September 2010 an. Denn eine nun mal bedeutende „,Realität“" sei ,die Zahl jener, die wir heute noch als Wehrpflichtige, als Wehrdienende haben" (beide Zitate: Deutscher Bundestag 2010d, S. 6106). Unter Verwendung des erneut auffälligen Schlüsselworts Realität führt er dabei als zentralen Grund für die damit einhergehende Wehrungerechtigkeit zunächst die stetige ,,Verkürzung der

\footnotetext{
25 Grund dafür war eine Plagiatsaffäre, die im Februar 2011 medienwirksam thematisiert wurde und schließlich zu dem Verlust seines Doktortitels sowie am 01. März 2011 zu seinem Rücktritt als Bundesminister der Verteidigung führte (vgl. z. B. ZEIT Online 2011b). Außerdem gab zu Guttenberg später alle weiteren politischen Ämter sowie sein Bundestagsmandat auf (vgl. Kulak et al. 2016, S. 16).

26 Das Bundeskabinett hatte die Wehrpflichtaussetzung sogar bereits am 15. Dezember 2010 beschlossen (vgl. Meyer 2010, S. 2; Kulak et al. 2016, S. 15).
} 
Grundwehrdienstdauer“" (ebd.) an ${ }^{27}$, sodass eine Verbindung zu seiner bundeswehrstrukturell-militärstrategischen Erzählstruktur ersichtlich wird.

Daran angekoppelt verknüpft er die Tatsache, dass insbesondere seit 2005 nur noch ein Bruchteil der Wehrpflichtigen tatsächlich zum Wehrdienst eingezogen wurde $^{28}$, mit der Frage, was denn denjenigen noch geboten werde, ,die zu uns kommen und zu uns kommen wollen und die für diesen unglaublich wichtigen Aspekt der Bindung zwischen Gesellschaft und Bundeswehr stehen“ (ebd.). Eine Antwort darauf, vor allem eine ,gesellschaftspolitisch[e] Begründung“, sei jedenfalls heute nur ,,schwierig“ (beide Zitate: ebd.) zu formulieren. Darüber hinaus sei die entscheidendste Frage: ,Ist für den einzelnen Wehrpflichtigen oder Wehrdienenden auch sicherheitspolitisch der Maßstab, den die Verfassung uns letztlich abverlangt, erfüllt? Diese Begründung können wir bereits heute nicht mehr in dem Maße geben“ (ebd.). Nach der Darlegung eines gesellschaftspolitischen Arguments zum Aus der Wehrpflicht bezieht sich der Verteidigungsminister nun mit diesen Erörterungen - sein sicherheitspolitisches Narrativmuster darin aufgreifend - also auch auf einen verfassungsrechtlichen Maßstab, wie ihn Mitte der 1990er-Jahre Roman Herzog betont hatte (siehe auch FN 18). Außerdem erhalten die Wehrpflichtigen von zu Guttenberg eine wichtige legitimationsbeschaffende Rolle als Helfer zugeschrieben, da er ihre Perspektive fortan zur Begründung seines Vorhabens nutzen wird. Indem er vorgibt, in ihrem Sinne zu handeln, wird die narrative Macht des Verteidigungsministers weiter gestärkt.

Diese Feststellung unterstützend lässt sich auch seine auf dem CDU-Parteitag im November 2010 gehaltene Rede anführen, in welcher zu Guttenberg noch ausführlicher seine verfassungsrechtlich-gesellschaftspolitische Argumentation entfaltet (vgl. CDU Deutschland 2010a, S. 132-133). Er spricht dort aber nicht nur über das „Stichwort Wehrgerechtigkeit“ (ebd., S. 132; Hervorh. d. Verf.) und die „verfassungsrechtliche Begründung“" (ebd., S. 133; Hervorh. d. Verf.) als wichtige Schlüsselkonzepte, sondern hält fest, dass ,[w]enn ein junger Mann heute nicht mehr zur Bundeswehr will [...], dann geht er dort auch nicht mehr hin“ (ebd., S. 132). Denn man wisse nun mal ,in jeder Schulklasse, wie man sich [...] drückt“ (ebd.). Dass die allgemeine Wehrpflicht keine Zukunft mehr habe, rechtfertigt der Minister demnach ebenfalls mit - zumindest von ihm wahrgenommenen - veränderten Prioritäten in der bundesdeutschen Gesellschaft - und wenn er einmal ,ganz ehrlich“ sei, dann habe er erkannt, dass ,,[d]er Wehrdienst und der Zivildienst [...] im Grunde mehr jun-

\footnotetext{
27 Tatsächlich stellte die Ausbildungszeit im Grundwehrdienst keinesfalls immer eine einheitliche Periode dar. Vielmehr gab es verschiedene Änderungen und im Zeitverlauf jeweils unterschiedliche Ausbildungszeiten: April 1957-März 1962: 12 Monate, April 1962-Juni 1962: 15 Monate, Juli 1962-Dezember 1972: 18 Monate, Januar 1973-September 1990: 15 Monate, Oktober 1990-Dezember 1995: 12 Monate, Januar 1996-Dezember 2001: 10 Monate, Januar 2002-Dezember 2010: 9 Monate, Januar 2011-Juni 2011: 6 Monate (vgl. Meyer 2010, S. II, 6, 17; Bundesregierung 2011).

28 Setzt man eine annehmbare Jahrgangsstärke mit ehemals durchschnittlich 400.000 Wehrpflichtigen in Deutschland (vgl. Tagesspiegel Online 2002; Kujat 2011, S. 4) ins Verhältnis zur Anzahl der zwischen den Jahren 1990 und 2009 in der Bundeswehr Grundwehrdienstleistenden und freiwillig länger Wehrdienstleistenden (vgl. Statista 2019), so ergibt sich folgendes Bild: Hatten in den Jahren 1990-1994 noch durchschnittlich knapp 46\% und zwischen 1995 und 1999 noch circa 40,6\% eines Jahrgangs im wehrpflichtigen Alter ihren Wehrdienst angetreten, so betrugen die Werte für die Jahre 2000-2004 nur noch etwa $29 \%$ bzw. für die Jahre 2005-2009 circa 17,2\%.
} 
ge Menschen zum Drückebergertum [verleiten] als dazu, dass sie tatsächlich einen sinnvollen Dienst machen“ (beide Zitate: ebd., S. 134).

Aus entsprechenden, mit der schon in den 1950er- und 1960er-Jahren im Wehrpflichtdiskurs abwertend verwendeten (Leit-)Metapher Drückebergertum (vgl. Steinbach 2011, S. 11, 15) aufgerufenen Gründen sei - so zu Guttenberg - eine politische Veränderung dringend notwendig. Diese halte er aber mit der Einrichtung eines zukünftig geplanten Freiwilligendienstes anstelle des verpflichtenden Wehr- oder Zivildienstes für realisierbar (vgl. CDU Deutschland 2010a, S. 135). Denn der neue Dienst biete nun eine echte Chance zur Etablierung einer neuen Kultur des individuellen Einsatzes und sei als Zeichen ,im Sinne einer aktiven Bürgergesellschaft“ (ebd., S. 136) zu verstehen (vgl. ebd.). Seine Formulierungen aktive Bürgergesellschaft durch einen ,freiwilligen Dienst an der Gesellschaft" (ebd., S. 135; Hervorh. d. Verf.) können in diesem Zusammenhang als weitere Schlüsselkonzepte herausgestellt werden, welche die Unionsmitglieder auf dem Parteitag davon überzeugen sollen, für die Wehrpflichtaussetzung zu stimmen. Schließlich - so lässt sich deuten - gebe es nur auf diese Weise die Möglichkeit für einen Wandel, der die Personen im wehrpflichtigen Alter wieder mehr in die gesellschaftliche Pflicht nehme.

Innerhalb einer gesamtgesellschaftlichen Stimmung, die heute kein ,freundliches Desinteresse“29 (BMVg 2010b, S. 13) mehr an der Bundeswehr zeige, sondern den Streitkräften mit „Neugier“ (Bundespresseamt 2010, S. 101) gegenüberstehe, sei so der Tenor zu Guttenbergs - ein freiwilliger Wehrdienst genau das Richtige. Allerdings müssten - wie er in der Bundestagsdebatte im Dezember 2010 erläutert - vor allem die ursprünglichen Gründungsnarrative der Bundeswehr für den neuen Freiwilligendienst weiter bestehen bleiben, da diese ,in den letzten Jahrzehnten mit dem Modell der Wehrpflicht eine Verstärkung“ (Deutscher Bundestag 2010f, S. 8843) erfahren hätten. Obwohl zu Guttenberg hier also den Stellenwert der Prinzipien rund um den Staatsbürger in Uniform anerkennt, schafft er es als homo narrans - wie schon in seiner Hamburger Grundsatzrede - erneut, die Bedenken, diese könnten bei einer Wehrpflichtaussetzung abgeschwächt werden, zu entkräften und die narrative Wirkkraft der Gründungsnarrative für eine Beibehaltung der Wehrpflicht als solche in den Hintergrund zu rücken. Denn diese sollen laut des Verteidigungsministers weiterhin ,maßgeblich“ bleiben sowie ,die künftigen Strukturen [...] wie [...] in den vergangenen Jahrzehnten prägen“" (beide Zitate: ebd.).

Insgesamt lässt sich sagen, dass der verfassungsrechtlich-gesellschaftspolitische Plot zum Ende des Jahres 2010 einen zentralen Stellenwert in den Argumentationsketten zu Guttenbergs einnimmt. Wenn der Minister im Hinblick auf die geschilderte Wehrungerechtigkeit auf dem CDU-Parteitag also an einer Stelle anmerkt, dass dies „nicht der maßgebliche Aspekt“ (CDU Deutschland 2010a, S. 132) für das Ende des Wehrdienstes sei, dann behält er damit nur insofern recht, als dass sein globalsicherheits- und außenpolitischer Plot mit einer noch größeren Sinnstiftungsstärke

\footnotetext{
29 Diese Formulierung geht zurück auf den ehemaligen Bundespräsidenten Horst Köhler (CDU), der diese in seiner Amtszeit gleich mehrmals gebraucht hatte, um festzustellen, dass die deutsche Bevölkerung zwar der Bundeswehr grundsätzlich vertraue, Aufmerksamkeit, Stolz oder Solidarität gegenüber den deutschen Streitkräften aber kaum gesellschaftlich vorherrsche (vgl. z. B. Bundespräsidialamt 2005, S. 6; Spiegel Online 2008).
} 

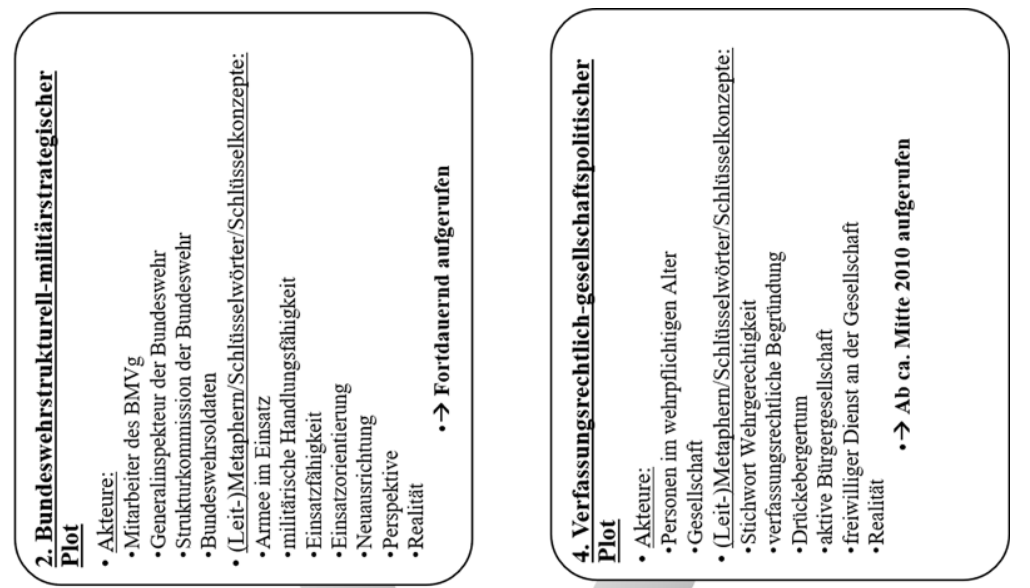

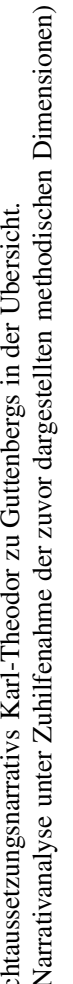

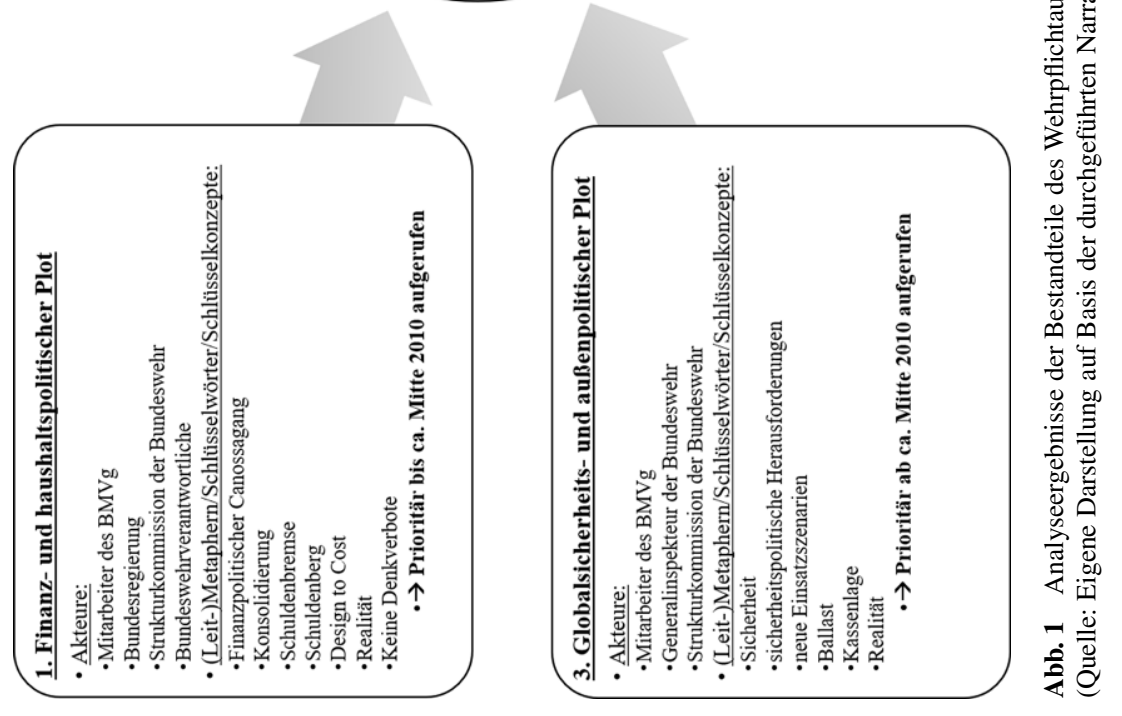


ausgestattet ist. Allerdings kann seine Relativierung wohl auch als parteipolitische Taktik interpretiert werden, die nicht nahelegen soll, den politischen Vorschlägen des Koalitionspartners gefolgt zu sein. Schließlich hatte die FDP schon in ihrem Bundestagswahlprogramm 2009 sowie unter anderem durch ein öffentlichkeitswirksames Presseinterview ihres damaligen Vorsitzenden, Guido Westerwelle, jenes Narrativmuster besetzt, das die Wehrpflicht als ungerecht und ihre Aufrechterhaltung deswegen als nicht mehr begründbar charakterisierte (vgl. Demmer 2009, S. 24; FDP 2009, S. 74; Meyer 2010, S. 1). Die Freien Demokraten müssen daher im diskursiv-polyphonen Kontext der Wehrpflicht-Debatte mindestens als eine auf die Erzählstrukturen zu Guttenbergs einwirkende Größe betrachtet werden. ${ }^{30}$

\section{Resümee und Ausblick}

Ziel des Beitrag war es, die Aussetzung der bundesdeutschen Wehrpflicht durch den ehemaligen Verteidigungsminister Karl-Theodor zu Guttenberg unter einem narrationsanalytischen Blickwinkel zu betrachten, um den damit verbundenen Politikwechsel jenseits der gängigen rationalistischen Forschungspostulate in der Politikwissenschaft mit einer erzähltheoretischen Deutung zu versehen. Dabei ließen sich vier verschiedene Erzählmuster kategorial identifizieren (siehe zusammenfassend Abb. 1), die durch den Akteur zu Guttenberg plotbildend in dessen Narrativ zur Legitimation des vollzogenen Policy-Wandels einbezogen wurden.

In der Gesamtschau lässt sich erkennen, dass Karl-Theodor zu Guttenberg als Sender seiner Erzählmuster einen ausschlaggebenden Einfluss auf die politisch vollzogenen Entscheidungen ausübte, die in einer Aussetzung - nicht Abschaffung - des Wehrdiensts mündeten. Als zentraler Akteur gelang es ihm schlussendlich, bis zum Ende des letzten Jahrzehnts in Deutschland diskursiv gültige Deutungen zur allgemeinen Wehrpflicht in den Hintergrund zu drängen und politisch wirkungsmächtig in einem polyphonen Kontext Zustimmung für einen ,historische[n] und gesellschaftliche[n] Bruch“ (Kujat 2011, S. 3) mit bis dahin existenten militärisch-kulturellen Traditionsbeständen der Bundesrepublik Deutschland zu generieren. Dazu konstruierte er ein sinnvermittelndes Gesamtnarrativ, das einen sequenziell strukturierten Ereigniszusammenhang auf der Grundlage komplexitätsreduzierender Erzählmuster aufwies und mit Gadinger et al. (2014c, S. 80) als ,eine sich hegemonialisierende Erzählung" beschrieben werden kann.

\footnotetext{
30 Ohnehin sei laut Meyer (vgl. 2010, S. 18) die durch die FDP eingebrachte diskursive Macht auf die Entscheidung zur Aussetzung der Wehrpflicht nicht zu unterschätzen. Wie er nämlich mutmaßt, bestehe die Möglichkeit, dass die FDP dem Kompromiss einer Wehrpflichtverkürzung auf sechs Monate im Rahmen der Koalitionsverhandlungen 2009 nur zugestimmt habe, ,weil sie hoffte, dass er all denjenigen, die Wehrpflichtige ausbilden müssen, vor eine Aufgabe stellte, die sie weder zur eigenen Zufriedenheit noch mit einem wirklichen Kompetenzgewinn für die Rekruten erfüllen konnten“ (ebd.). Wäre dem so, dann hätte die FDP - so Meyer weiter - die Wehrdienstverkürzung genutzt, ,um denjenigen, die bisher die Wehrpflicht für unverzichtbar hielten, die Augen dafür zu öffnen, dass es eine nicht unterschreitbare Grenze bei der Anpassung der Dauer des Grundwehrdienstes an die Erfordernisse der Wehrgerechtigkeit gibt. Dann wäre das Ende Juni 2010 beschlossene Wehrrechtsänderungsgesetz 2010 so etwas wie ein Dummy für den Crashtest der Wehrpflicht gewesen“ (ebd.).
} 
Werden mögliche Erfolgsfaktoren des Wehrpflichtaussetzungsnarrativs reflektiert, so ist die Beobachtung von Bedeutung, dass der politische Erfolg einer Erzählung nicht nur durch eine ,strukturiert[e] Kraft“ oder dem Merkmal, „Ressourcen des Kulturellen oder Diskursiven für sich vereinnahmen zu können“ (beide Zitate: Gadinger et al. 2014b, S. 34), bedingt ist. Stattdessen ist es zusätzlich ein gewisses $\mathrm{Maß}$ an „Flexibilität und [...] ihr[e] darin begründet[e] Fähigkeit, sich an überraschende Wendungen rasch und plausibel anpassen zu können“ (ebd.), die eine entscheidende Variable darstellt. Denn als Verteidigungsminister war zu Guttenberg nicht nur in der (macht-)politischen Position, die Wehrpflichtaussetzung auf die politische Agenda und den politischen Entscheidungsweg zu bringen, sondern sein dazu benutztes Narrativ offenbart in Gänze auch ein ausgeprägtes Anpassungsvermögen. Darüber hinaus charakterisiert sich seine entfaltete Erzählung im Hinblick auf die Ausgestaltung der darin kategorisierbaren Plots zum einen durch eine im Zeitverlauf unterschiedliche Verwendung von hochfrequenten (Leit-)Metaphern und Schlüsselwörtern. Zum anderen lassen sich aber auch spezifisch zugewiesene Akteurskonstellationen finden, die für seine Narration konstitutiv sind. So erwähnte zu Guttenberg eine Vielzahl von Helfern, die seine Narrativmuster zwar meist nicht proaktiv unterstützten, welche allerdings durch den Verteidigungsminister als unterstützende Legitimationsinstanzen in seine Narrativstrukturen einbezogen wurden. Ebenso adressierte der Minister mit seinen narrativen Mustern neben der breiten Öffentlichkeit einige ausgewählte Empfänger, die er als Rezipienten seiner Story intendierte.

Dass Karl-Theodor zu Guttenberg letztlich zum Helden seiner eigenen Geschichte wurde, lässt sich - jenseits der Stärke und Dynamik seiner Narration - allerdings auch auf die kommunikativen (Führungs-)Kompetenzen seiner Person zurückführen, sodass diese ebenfalls als akteurzentrierte Gelingensbedingung für die Erfolgsaussichten seiner Reforminitiative bedacht werden müssen (vgl. auch Harnisch und Weiß 2014, S. 228-231, 235). Sein durchweg mithilfe seiner Plotstrukturen selbst konstruiertes Bild als pragmatischer Politiker sowie sein Auftreten mit stets reformerischem Duktus, das die CDU/CSU mit einem „Guttenberg-Virus“ (Denkler 2010) infizierte und ihm eine hohe Popularität in der Bevölkerung sicherte (vgl. Kulak et al. 2016, S. 14) ${ }^{31}$, stattete ihn dabei mit einem großen öffentlichkeitswirksamen Impetus aus. Sehr wahrscheinlich ist, dass diese Eigenschaften des homo narrans schlussendlich - zusätzlich zu den entfalteten Narrativmustern - mitentscheidend halfen, das politische Anliegen des Wehrpflichtendes durchzusetzen. ${ }^{32}$ Auf dieser

\footnotetext{
31 Laut ARD-DeutschlandTREND von Infratest dimap (vgl. 2010, S. 12) waren im Oktober $201074 \%$ der Befragten mit der politischen Arbeit zu Guttenbergs zufrieden oder sehr zufrieden (Platz 1), nur $22 \%$ zeigten sich weniger oder gar nicht zufrieden und nur $4 \%$ gaben an, ihn nicht zu kennen oder kein Urteil abgeben zu wollen. Im Vergleich dazu lagen beispielsweise die Zufriedenheitswerte für Angela Merkel bei nur $41 \%$ (Platz 8) und für Horst Seehofer bei nur 33\% (Platz 9).

32 Diese Perspektive verstärkend liegt die Vermutung nahe, dass der beispielsweise im Sommer 2010 durch das Meinungsforschungsinstitut Emnid aus der Bevölkerung heraus gemessene Zustimmungswert zur Wehrpflichtaussetzung von 63\% der damals Befragten (vgl. Welt Online 2010) insgesamt mehr auf die in der Bevölkerung ausgeprägte Beliebtheit des Verteidigungsministers als auf eine inhaltliche Überzeugung für den Politikwechsel zurückgeführt werden kann - zumal sich noch in den Jahren zuvor, ,[i]m Hinblick auf die beiden Alternativen Beibehaltung oder Abschaffung der Wehrpflicht [...] zwei von drei Bundesbürgern für die Wehrpflicht [aussprachen]“ (Werkner 2011, S. 44).
} 
Grundlage kann zudem argumentiert werden, dass sowohl die durch zu Guttenberg vollzogenen Veränderungen der narrativen Begründungszusammenhänge als auch sein charakteristisches Auftreten am Ende sogar ein Verblassen der Relevanz anfänglicher politischer Versprechungen ermöglichten. Denn das durch den Minister zu Beginn seiner Initiative betonte finanz- bzw. haushaltspolitische Narrativmuster stellte sich rückblickend als argumentative Hülse heraus, die nicht der politischen Realität entsprach.

Alles in allem vermag dieser Beitrag anhand des untersuchten Fallbeispiels exemplarisch zu zeigen, dass politische Veränderungen häufig an Voraussetzungen einer (akteurzentrierten) erzählerischen Artikulation geknüpft sind, sodass die Untersuchung von politischen Narrativen - zumindest in der interpretativen PolicyForschung - einen wichtigen Mehrwert bietet. Denn eine auf die politische Agenda gerückte Idee, welche dann die Entscheidungsprozesse der Politik beeinflusst, ist nicht selten ein Ergebnis der Gestaltung von narrativen Mustern. Auf diese Weise nehmen politische Akteure Einfluss auf die Durchsetzung von Policy-Veränderungen, deren Schnelligkeit sowie Erfolg letztendlich an den legitimatorischen Grad der Sinnstiftung und Überzeugungskraft sowie Glaubwürdigkeit und Dringlichkeitsbetonung geknüpft ist. Somit sollten narrationsanalytische Untersuchungen in der Politikwissenschaft durchgeführt werden, um jenseits kausalanalytischer Forschungsprämissen politische Aushandlungsprozesse in ihrer gesamten Komplexität beschreiben zu können.

Funding Open Access funding provided by Projekt DEAL.

Open Access Dieser Artikel wird unter der Creative Commons Namensnennung 4.0 International Lizenz veröffentlicht, welche die Nutzung, Vervielfältigung, Bearbeitung, Verbreitung und Wiedergabe in jeglichem Medium und Format erlaubt, sofern Sie den/die ursprünglichen Autor(en) und die Quelle ordnungsgemäß nennen, einen Link zur Creative Commons Lizenz beifügen und angeben, ob Änderungen vorgenommen wurden.

Die in diesem Artikel enthaltenen Bilder und sonstiges Drittmaterial unterliegen ebenfalls der genannten Creative Commons Lizenz, sofern sich aus der Abbildungslegende nichts anderes ergibt. Sofern das betreffende Material nicht unter der genannten Creative Commons Lizenz steht und die betreffende Handlung nicht nach gesetzlichen Vorschriften erlaubt ist, ist für die oben aufgeführten Weiterverwendungen des Materials die Einwilligung des jeweiligen Rechteinhabers einzuholen.

Weitere Details zur Lizenz entnehmen Sie bitte der Lizenzinformation auf http://creativecommons.org/ licenses/by/4.0/deed.de. 
Anhang

Tab. 2 Übersicht der Reden Karl-Theodor zu Guttenbergs während seiner Amtszeit als Verteidigungsminister mit Themenbezug Aussetzung der Wehrpflicht vor dem 17. Deutschen Bundestag

\begin{tabular}{ll}
\hline Datum & Anlass und Thema \\
\hline 20.01.2010 & $\begin{array}{l}\text { Rede zur „Erste[n] Beratung des von der Bundesregierung eingebrachten Entwurfs ei- } \\
\text { nes Gesetzes über die Feststellung des Bundeshaushaltsplans für das Haushaltsjahr } 2010 \\
\text { (Haushaltsgesetz 2010)“ (Deutscher Bundestag 2010a) }\end{array}$ \\
19.05.2010 & $\begin{array}{l}\text { Rede/Bericht bei der Befragung der Bundesregierung zum „Gesetzentwurf zur Änderung } \\
\text { wehr- und zivildienstrechtlicher Vorschriften“ (Deutscher Bundestag 2010b) }\end{array}$ \\
11.06.2010 & $\begin{array}{l}\text { Rede zur „Erste[n] Beratung des von den Fraktionen der CDU/CSU und der FDP ein- } \\
\text { gebrachten Entwurfs eines Gesetzes zur Änderung wehr- und zivildienstrechtlicher Vor- } \\
\text { schriften 2010 (Wehrrechtsänderungsgesetz 2010 - WehrRÄndG 2010)“ (Deutscher Bun- } \\
\text { destag 2010c) }\end{array}$ \\
15.09.2010 & $\begin{array}{l}\text { Rede zur „Erste[n] Beratung des von der Bundesregierung eingebrachten Entwurfs ei- } \\
\text { nes Gesetzes über die Feststellung des Bundeshaushaltsplans für das Haushaltsjahr 2011 } \\
\text { (Haushaltsgesetz 2011)“ (Deutscher Bundestag 2010d) }\end{array}$ \\
24.11.2010 & $\begin{array}{l}\text { Rede zur „ZWweite[n] Beratung des von der Bundesregierung eingebrachten Entwurfs ei- } \\
\text { nes Gesetzes über die Feststellung des Bundeshaushaltsplans für das Haushaltsjahr 2011 } \\
\text { (Haushaltsgesetz 2011)“ (Deutscher Bundestag 2010e) }\end{array}$ \\
15.12.2010 & $\begin{array}{l}\text { Rede/Bericht bei der Befragung der Bundesregierung zum „Entwurf eines Gesetzes zur } \\
\text { Änderung wehrrechtlicher Vorschriften 2011 und Entwurf eines Gesetzes zur Einführung } \\
\text { eines Bundesfreiwilligendienstes“ (Deutscher Bundestag 2010f) } \\
\text { Rede zur „Erste[n] Beratung des von der Bundesregierung eingebrachten Entwurfs eines } \\
\text { Gesetzes zur Änderung wehrrechtlicher Vorschriften 2011 (Wehrrechtsänderungsgesetz } \\
\text { 2011 - WehrRÄndG 2011)“(Deutscher Bundestag 2011) }\end{array}$ \\
\hline
\end{tabular}

Quelle: Eigene Darstellung; Hinweis: Alle Reden wurden auf der Basis der jeweils vorliegenden stenographischen Berichte (Plenarprotokolle) des Deutschen Bundestages analysiert.

Tab. 3 Übersicht der ausgewählten Reden Karl-Theodor zu Guttenbergs während seiner Amtszeit als Verteidigungsminister mit Themenbezug Aussetzung der Wehrpflicht außerhalb des Deutschen Bundestages

\begin{tabular}{ll}
\hline Datum & Anlass und Thema \\
\hline 26.05 .2010 & $\begin{array}{l}\text { Grundsatzrede anlässlich des Besuchs der Führungsakademie der Bundeswehr und der } \\
\text { Kommandeurtagung der Streitkräftebasis in Hamburg (vgl. BMVg 2010a) }\end{array}$ \\
04.09.2010 & $\begin{array}{l}\text { Rede anlässlich der Fachkonferenz Zukunft Bundeswehr }- \text { eine gesamtgesellschaftliche } \\
\text { Herausforderung der Frauenunion Bayern in Kulmbach (vgl. BMVg 2010b) }\end{array}$ \\
$\begin{array}{l}\text { 15.11.2010 } \\
\text { Rede beim CDU-Parteitag in Karlsruhe (vgl. CDU Deutschland 2010a) }\end{array}$ \\
\hline
\end{tabular}

Quelle: Eigene Darstellung; Hinweis: Alle Reden wurden auf der Basis vorliegender, archivierter Textdokumente analysiert. Im Sinne einer Quellenkritik galt vor Ort das gesprochene Wort. 


\section{Literatur}

Barbehön, Marlon, und Sybille Münch. 2014. Die Stadt als Sinnhorizont: Zur Kontextgebundenheit politischer Narrative. In Politische Narrative. Konzepte - Analysen - Forschungspraxis, Hrsg. Frank Gadinger, Sebastian Jarzebski, und Taylan Yildiz, 149-171. Wiesbaden: Springer VS.

Biegoń, Dominika, und Frank Nullmeier. 2014. Narrationen über Narrationen. Stellenwert und Methodologie der Narrationsanalyse. In Politische Narrative. Konzepte - Analysen - Forschungspraxis, Hrsg. Frank Gadinger, Sebastian Jarzebski, und Taylan Yildiz, 39-65. Wiesbaden: Springer VS.

BMF (Bundesministerium der Finanzen). 2010. Bundeshaushaltsplan 2010. Einzelplan 14. Bundesministerium der Verteidigung. https://www.bundeshaushalt.de/fileadmin/de.bundeshaushalt/content_de/ dokumente/2010/Bundeshaushaltsplan_2010.zip. Zugegriffen: 16. Okt. 2019.

BMF (Bundesministerium der Finanzen). 2011. Bundeshaushaltsplan 2011. Einzelplan 14. Bundesministerium der Verteidigung. https://www.bundeshaushalt.de/fileadmin/de.bundeshaushalt/content_de/ dokumente/2011/Bundeshaushaltsplan_2011.zip. Zugegriffen: 16. Okt. 2019.

BMF (Bundesministerium der Finanzen). 2012. Bundeshaushaltsplan 2012. Einzelplan 14. Bundesministerium der Verteidigung. https://www.bundeshaushalt.de/fileadmin/de.bundeshaushalt/content_de/ dokumente/2012/soll/Haushaltsplan-2012.pdf. Zugegriffen: 16. Okt. 2019.

BMF (Bundesministerium der Finanzen). 2013. Bundeshaushaltsplan 2013. Einzelplan 14. Bundesministerium der Verteidigung. https://www.bundeshaushalt.de/fileadmin/de.bundeshaushalt/content_de/ dokumente/2013/soll/Haushaltsplan-2013.pdf. Zugegriffen: 16. Okt. 2019.

BMF (Bundesministerium der Finanzen). 2014. Bundeshaushaltsplan 2014. Einzelplan 14. Bundesministerium der Verteidigung. https://www.bundeshaushalt.de/fileadmin/de.bundeshaushalt/content_de/ dokumente/2014/soll/Haushaltsplan-2014.pdf. Zugegriffen: 16. Okt. 2019.

BMF (Bundesministerium der Finanzen). 2015. Bundeshaushaltsplan 2015. Einzelplan 14. Bundesministerium der Verteidigung. https://www.bundeshaushalt.de/fileadmin/de.bundeshaushalt/content_de/ dokumente/2015/soll/Haushaltsplan-2015.pdf. Zugegriffen: 16. Okt. 2019.

BMF (Bundesministerium der Finanzen). 2016. Bundeshaushaltsplan 2016. Einzelplan 14. Bundesministerium der Verteidigung. https://www.bundeshaushalt.de/fileadmin/de.bundeshaushalt/content_de/ dokumente/2016/soll/Haushaltsplan-2016.pdf. Zugegriffen: 16. Okt. 2019.

BMF (Bundesministerium der Finanzen). 2017. Bundeshaushaltsplan 2017. Einzelplan 14. Bundesministerium der Verteidigung. https://www.bundeshaushalt.de/fileadmin/de.bundeshaushalt/content_de/ dokumente/2017/soll/Gesamt_Haushalt_2017_mit_HG.pdf. Zugegriffen: 16. Okt. 2019.

BMF (Bundesministerium der Finanzen). 2018. Bundeshaushaltsplan 2018. Einzelplan 14. Bundesministerium der Verteidigung. https://www.bundeshaushalt.de/fileadmin/de.bundeshaushalt/content_de/ dokumente/2018/soll/Bundeshaushaltsplan-2018-Haushaltsgesetz-2018.pdf. Zugegriffen: 16. Okt. 2019.

BMF (Bundesministerium der Finanzen). 2019. Bundeshaushaltsplan 2019. Einzelplan 14. Bundesministerium der Verteidigung. https://www.bundeshaushalt.de/fileadmin/de.bundeshaushalt/content_ de/dokumente/2019/soll/Haushaltsgesetz_2019_Bundeshaushaltsplan_Gesamt.pdf. Zugegriffen: 16. Okt. 2019.

BMVg (Bundesministerium der Verteidigung). 2010a. Grundsatzrede des Bundesministers der Verteidigung, Dr. Karl-Theodor Freiherr zu Guttenberg, anlässlich des Besuches der Führungsakademie der Bundeswehr und der Kommandeurtagung der Streitkräftebasis am 26. Mai 2010 in Hamburg. Unkorrigierte Abschrift. Es gilt das gesprochene Wort.

BMVg (Bundesministerium der Verteidigung). 2010b. Rede des Bundesministers der Verteidigung, Dr. Karl-Theodor Freiherr zu Guttenberg, anlässlich der Fachkonferenz der Frauenunion Bayern ,Zukunft Bundeswehr - eine gesamtgesellschaftliche Herausforderung“ am 4. September 2010 in Kulmbach. Es gilt das gesprochene Wort.

Bredow, Wilfried von. 2015. Sicherheit, Sicherheitspolitik und Militär. Deutschland seit der Vereinigung. Wiesbaden: Springer VS.

Bundespräsidialamt. 2005. Einsatz für Freiheit und Sicherheit. Rede von Bundespräsident Horst Köhler bei der Kommandeurtagung der Bundeswehr am 10. Oktober 2005 in Bonn. http://www. bundespraesident.de/SharedDocs/Reden/DE/Horst-Koehler/Reden/2005/10/20051010_Rede.html. Zugegriffen: 24. März 2020.

Bundespresseamt (Presse- und Informationsamt der Bundesregierung). 2010. Stichworte zur Sicherheitspolitik. Nr. 11/12. November/Dezember 2010. https://www.bundesregierung.de/resource/blob/ 976072/760144/e1f9e829128\%20a1743d9074ab0d3e84b6e/2011-01-19-sipo-november-dezember2010-data.pdf?download=1. Zugegriffen: 19. Aug. 2019. 
Bundesregierung. 2011. Freiwilliger Wehrdienst - Chance statt Pflicht. https://archiv.bundesregierung.de/ archiv-de/freiwilliger-wehrdienst-chance-statt-pflicht-425212. Zugegriffen: 2. Okt. 2019.

CDU Deutschland. 2010a. Protokoll. 23. Parteitag der CDU Deutschlands. 15.-16. November 2010, Karlsruhe. https://www.kas.de/c/document_library/get_file?uuid=09629588-3aba-870d-c53824131d21378d\&groupId=252038. Zugegriffen: 8. Okt. 2019.

CDU Deutschland. 2010b. Zukunft der Bundeswehr. Beschluss des 23. Parteitages der CDU Deutschlands. https://www.kas.de/c/document_library/get_file?uuid=94abcc97-1152-e97a-6c15-17ce1031c127\& groupId=252038. Zugegriffen: 8. Okt. 2019.

CDU, CSU, und FDP. 2009. Wachstum. Bildung. Zusammenhalt. Der Koalitionsvertrag zwischen CDU, CSU und FDP. 17. Legislaturperiode. https://www.kas.de/c/document_library/get_file? uuid=83dbb842-b2f7-bf99-6180-e65b2de7b4d4\&groupId=252038. Zugegriffen: 24. Juni 2020.

Demmer, Ulrike. 2009. Überflüssig und ungerecht. Der Spiegel 63(31):24.

Denkler, Thorsten. 2010. Guttenberg missioniert die CDU. https://www.sueddeutsche.de/politik/cdustimmt-fuer-aussetzung-der-wehrpflicht-guttenberg-missioniert-die-cdu-1.1024216. Zugegriffen: 28. Okt. 2019.

Der Bundeswahlleiter. 2020. Bundestagswahl 2009. Wahl zum 17. Deutschen Bundestag am 27. September 2009. https://www.bundeswahlleiter.de/bundestagswahlen/2009.html. Zugegriffen: 22. Juli 2020.

Deutscher Bundestag. 2010a. Stenographischer Bericht. 15. Sitzung. Plenarprotokoll 17/15 (20.01.2010). http://dipbt.bundestag.de/doc/btp/17/17015.pdf. Zugegriffen: 19. Aug. 2019.

Deutscher Bundestag. 2010b. Stenographischer Bericht. 42. Sitzung. Plenarprotokoll 17/42 (19.05.2010). http://dip21.bundestag.de/dip21/btp/17/17042.pdf. Zugegriffen: 19. Aug. 2019.

Deutscher Bundestag. 2010c. Stenographischer Bericht. 47. Sitzung. Plenarprotokoll 17/47 (11.06.2010). http://dip21.bundestag.de/dip21/btp/17/17047.pdf. Zugegriffen: 19. Aug. 2019.

Deutscher Bundestag. 2010d. Stenographischer Bericht. 58. Sitzung. Plenarprotokoll 17/58 (15.09.2010). http://dipbt.bundestag.de/doc/btp/17/17058.pdf. Zugegriffen: 19. Aug. 2019.

Deutscher Bundestag. 2010e. Stenographischer Bericht. 74. Sitzung. Plenarprotokoll 17/74 (24.11.2010). http://dipbt.bundestag.de/dip21/btp/17/17074.pdf. Zugegriffen: 19. Aug. 2019.

Deutscher Bundestag. 2010f. Stenographischer Bericht. 80. Sitzung. Plenarprotokoll 17/80 (15.12.2010). http://dipbt.bundestag.de/dip21/btp/17/17080.pdf. Zugegriffen: 19. Aug. 2019.

Deutscher Bundestag. 2011. Stenographischer Bericht. 93. Sitzung. Plenarprotokoll 17/93 (24.02.2011). http://dip21.bundestag.de/dip21/btp/17/17093.pdf. Zugegriffen: 19. Aug. 2019.

van Eeten, Michel J.G. 2007. 18. Narrative policy analysis. In Handbook of public policy analysis. Theory, politics, and methods, Hrsg. Frank Fischer, Gerald J. Miller, und Mara S. Sidney, 251-269. Boca Raton: CRC Press.

FDP. 2009. Die Mitte stärken. Deutschlandprogramm 2009. Programm der Freien Demokratischen Partei zur Bundestagswahl 2009. Beschlossen auf dem Bundesparteitag vom 15.-17. Mai 2009 in Hannover. https://www.fdp.de/files/565/Deutschlandprogramm09_Endfassung.pdf. Zugegriffen: 24. Juni 2020.

Fisher, Walter R. 1985. Homo Narrans. The narrative paradigm: in the beginning. Journal of Communication 35(4):74-89.

Florack, Martin, Timo Grunden, und Karl-Rudolf Korte. 2015. Regierungsorganisation und Kernexekutive: Thesen zu einer modernen Regierungsforschung. Zeitschrift für Politikwissenschaft (ZPol) 25(4):617-634.

Frankfurter Allgemeine Zeitung Online. 2010a. Guttenberg: Keine Sicherheitspolitik nach Kassenlage. https://www.faz.net/aktuell/politik/inland/wehrpflicht-wird-ausgesetzt-guttenberg-keinesicherheitspolitik-nach-kassenlage-1579347.html. Zugegriffen: 24. Okt. 2019.

Frankfurter Allgemeine Zeitung Online. 2010b. Kurswechsel in der Finanzpolitik geschafft. https:// www.faz.net/aktuell/wirtschaft/wirtschaftspolitik/haushalt-2011-verabschiedet-kurswechsel-in-derfinanzpolitik-geschafft-11066763.html. Zugegriffen: 17. Okt. 2019.

Gadinger, Frank. 2019. Lügenpresse, gesunder Volkskörper, tatkräftiger Macher: Erzählformen des Populismus. In Narrative des Populismus. Erzählmuster und -strukturen populistischer Politik, Hrsg. Michael Müller, Jørn Precht, 115-146. Wiesbaden: Springer VS.

Gadinger, Frank, und Elena Simon. 2019. Kalkulierte Ambivalenz, mobilisierte Ängste und volksnahe Inszenierung: Rechtspopulistische Erzählstrategien in Wahlkampagnen und Regierungspraxis. Zeitschrift für Politikwissenschaft (ZPol) 29(1):23-52.

Gadinger, Frank, und Taylan Yildiz. 2017. 25 Politik. In Erzählen. Ein interdisziplinäres Handbuch, Hrsg. Matías Martínez, 158-165. Stuttgart: J.B. Metzler.

Gadinger, Frank, Sebastian Jarzebski, und Taylan Yildiz (Hrsg.). 2014a. Politische Narrative. Konzepte Analysen - Forschungspraxis. Wiesbaden: Springer VS. 
Gadinger, Frank, Sebastian Jarzebski, und Taylan Yildiz. 2014b. Politische Narrative. Konturen einer politikwissenschaftlichen Erzähltheorie. In Politische Narrative. Konzepte - Analysen - Forschungspraxis, Hrsg. Frank Gadinger, Sebastian Jarzebski, und Taylan Yildiz, 3-38. Wiesbaden: Springer VS.

Gadinger, Frank, Sebastian Jarzebski, und Taylan Yildiz. 2014c. Vom Diskurs zur Erzählung. Möglichkeiten einer politikwissenschaftlichen Narrativanalyse. Politische Vierteljahresschrift (PVS) 55(1):67-93.

Gadinger, Frank, Christopher Smith Ochoa, und Taylan Yildiz. 2019. Resistance or thuggery? Political narratives of urban riots. Narrative Culture 6(1):88-111.

Gadinger, Frank, Sebastian Jarzebski, und Taylan Yildiz. 2015. Progressive Politik in pragmatischen Zeiten: Politische Narrative gesellschaftlichen Wandels. Werkbericht Nr. 6. Forschungsgruppe „Politische Narrative“. https:/www.boell.de/sites/default/files/2015-07-progressive-politik-inpragmatischen-zeiten.pdf. Zugegriffen: 13. Aug. 2019.

Gottweis, Herbert. 2003. Post-positivistische Zugänge in der Policy-Forschung. In Politik als Lernprozess. Wissenszentrierte Ansätze in der Politikanalyse, Hrsg. Matthias Leonhard Maier, Achim Hurrelmann, Frank Nullmeier, Tanja Pritzlaff, und Achim Wiesner, 122-138. Opladen: Leske + Budrich.

Graça Peters, Katharina. 2010. Guttenbergs Sparpläne verärgern Parteifreunde. https://www.spiegel.de/ politik/deutschland/bundeswehrreform-guttenbergs-sparplaene-veraergern-parteifreunde-a- 698578 . html. Zugegriffen: 15. Juli 2020.

Groß, Jürgen, und Dieter S. Lutz (Hrsg.). 1998. Wehrpflicht ausgedient. Baden-Baden: Nomos.

Harnisch, Sebastian, und Simon Weiß. 2014. Rapider Politikwechsel in der deutschen Verteidigungspolitik: eine analytische Kurzgeschichte der Suspendierung der Wehrpflicht. In Rapide Politikwechsel in der Bundesrepublik. Theoretischer Rahmen und empirische Befunde, Hrsg. Friedbert W. Rüb, 205-236. Baden-Baden: Nomos.

Hartleb, Florian. 2012. Karl-Theodor zu Guttenberg: Inszenierung bis zur Implosion. Zeitschrift für Politikberatung (ZPB) 5(1):34-38.

Hofmann, Wilhelm, Judith Renner, und Katja Teich (Hrsg.). 2014. Narrative Formen der Politik. Wiesbaden: Springer VS.

Infratest dimap. 2010. ARD-DeutschlandTREND. Oktober 2010. Eine Umfrage zur politischen Stimmung im Auftrag der ARD-Tagesthemen und fünf Tageszeitungen. https://www.infratest-dimap.de/ fileadmin/_migrated/content_uploads/dt1010_bericht.pdf. Zugegriffen: 29. Okt. 2019.

Jarzebski, Sebastian. 2015. Wahlkampf als Erzählung: Metaphern und Narrative im TV-Duell. In Die Bundestagswahl 2013. Analysen der Wahl-, Parteien-, Kommunikations- und Regierungsforschung, Hrsg. Karl-Rudolf Korte, 367-385. Wiesbaden: Springer VS.

Kaplan, Thomas J. 1993. Reading policy narratives: beginnings, middles and ends. In The argumentative turn in policy analysis and planning, Hrsg. Frank Fischer, John Forester, 167-185. Durham/London: Duke University Press.

Koschorke, Albrecht. 2013. Wahrheit und Erfindung. Grundzüge einer allgemeinen Erzähltheorie, 3. Aufl., Frankfurt am Main: S. Fischer.

Kujat, Harald. 2011. Das Ende der Wehrpflicht. Essay. Aus Politik und Zeitgeschichte (APuZ) 61(48):3-7.

Kulak, Anna, Timo Schummers, Daniel Reichard, und Alexander Geiger. 2016. Guttenbergs Meisterstück? Die Aussetzung der Wehrpflicht. Überarbeitete Fassung - Stand 16.01.2018. https://www. uni-koblenz-landau.de/de/landau/fb6/sowi/pw/abteilung/politische-system-brd/caseteaching/copy_ of_Kulaketal.2016_Case_AussetzungderWehrpflicht.pdf/view. Zugegriffen: 24. März 2020.

Lakoff, George, und Mark Johnson. 2008. Leben in Metaphern. Konstruktion und Gebrauch von Sprachbildern, 6. Aufl., Heidelberg: Auer.

Lohse, Eckart, und Markus Wehner. 2011. Guttenberg. Biographie, 2. Aufl., München: Droemer.

Löwenstein, Stephan, und Albert Schäffer. 2011. Eine seltene Erscheinung. https://www.faz.net/aktuell/ politik/die-guttenberg-affaere/der-politiker-zu-guttenberg-eine-seltene-erscheinung-15023/28-102009-guttenberg-wird-1616126.html. Zugegriffen: 15. Nov. 2019.

Meyer, Berthold. 2010. Bundeswehr ohne Wehrpflichtige - Was folgt daraus für die Parlamentsarmee im Einsatz? HSFK-Report Nr. 11/2010. https:/www.hsfk.de/fileadmin/HSFK/hsfk_downloads/ report1110.pdf. Zugegriffen: 24. März 2020.

Müller, Michael, und Jørn Precht (Hrsg.). 2019. Narrative des Populismus. Erzählmuster und -strukturen populistischer Politik. Wiesbaden: Springer VS.

Münch, Sybille. 2016. Interpretative Policy-Analyse. Eine Einführung. Wiesbaden: Springer VS.

Oppermann, Kai, und Alexander Spencer. 2016. „Es war einmal ein Fehler...“: Fiaskos als Narrative in der Außenpolitik. Zeitschrift für Politik (ZfP) 63(2):156-169. 
Oppermann, Kai, und Alexander Spencer. 2018. Narrating success and failure: congressional debates on the 'Iran nuclear deal'. European Journal of International Relations 24(2):268-292.

Raidel, Hans. 1998. Die Bundeswehr. Grundlagen, Rollen, Aufgaben. München: Akademie für Politik und Zeitgeschehen.

Roe, Emery. 1994. Narrative policy analysis. Theory and practice. Durham/London: Duke University Press.

Rose, Jürgen. 1998. Die prekär gewordene Legitimation der Wehrpflicht. In Wehrpflicht ausgedient, Hrsg. Jürgen Groß, Dieter S. Lutz, 31-62. Baden-Baden: Nomos.

Schmidt, Michael. 2010. Der Wettkampf um die Reformmodelle hat begonnen. https://www.zeit.de/ politik/deutschland/2010-08/wehrpflicht-bundeswehr-guttenberg/komplettansicht. Zugegriffen: 24 . Okt. 2019.

Schneider, Volker, und Frank Janning. 2006. Politikfeldanalyse. Akteure, Diskurse und Netzwerke in der öffentlichen Politik. Wiesbaden: VS.

Seidler, Franz W., und Helmut Reindl. 1973. Wehrpflicht, Kriegsdienstverweigerung, Zivildienst, Wehrgerechtigkeit. Bonn: Bundeszentrale für politische Bildung.

Spencer, Alexander. 2016. Romantic narratives in international politics. Pirates, rebels and mercenaries. Manchester: Manchester University Press.

Spencer, Alexander. 2019. Narratives and the romantic genre in IR: dominant and marginalized stories of Arab rebellion in Libya. International Politics 56(1):123-140.

Spiegel Online. 2008. Köhler fordert mehr Aufklärung über Auslandseinsätze. https://www.spiegel. $\mathrm{de} /$ politik/deutschland/bundeswehr-koehler-fordert-mehr-aufklaerung-ueber-auslandseinsaetze-a593131.html. Zugegriffen: 17. Okt. 2019.

Spiegel Online. 2010a. Merkel hält Aussetzen der Wehrpflicht für möglich. https://www.spiegel.de/politik/ deutschland/umdenken-in-der-union-merkel-haelt-aussetzen-der-wehrpflicht-fuer-moeglich-a700044.html. Zugegriffen: 11. Okt. 2019.

Spiegel Online. 2010b. Union probt Aufstand gegen Verteidigungsminister. https://www.spiegel.de/politik/ deutschland/zoff-um-wehrpflicht-union-probt-aufstand-gegen-verteidigungsminister-a-708250. html. Zugegriffen: 20. Juli 2020.

Spiegel Online. 2011. Guttenberg geht, de Maizière kommt. https://www.spiegel.de/politik/deutschland/ verteidigungsministerium-guttenberg-geht-de-maiziere-kommt-a-748804.html. Zugegriffen: 15. Okt. 2019.

Statista. 2019. Anzahl der Grundwehrdienstleistenden und freiwillig länger Dienstleistenden in der Bundeswehr nach dem Jahr des Diensteintritts von 1990 bis 2009. https://de.statista.com/statistik/daten/ studie/152202/umfrage/entwicklung-der-anzahl-der-wehrdienstleistenden-in-der-bundeswehr-seit1990/. Zugegriffen: 2. Okt. 2019.

Steinbach, Peter. 2011. Zur Geschichte der Wehrpflicht. Essay. Aus Politik und Zeitgeschichte (APuZ) 61(48):8-15.

Stierstorfer, Klaus. 2013. Linguistic turn. In Metzler Lexikon Literatur- und Kulturtheorie. Ansätze - Personen-Grundbegriffe, 5. Aufl., Hrsg. Ansgar Nünning, 447-448. Stuttgart/Weimar: J.B. Metzler.

Stone, Deborah A. 1989. Causal stories and the formation of policy agendas. Political Science Quarterly 104(2):281-300.

Strukturkommission der Bundeswehr. 2010. Bericht der Strukturkommission der Bundeswehr. Oktober 2010. Vom Einsatz her denken. Konzentration, Flexibilität, Effizienz. https://www.roderichkiesewetter.de/fileadmin/Service/Dokumente/20101026-weise-kommisionsbericht.pdf. Zugegriffen: 10. Okt. 2019.

Süddeutsche Zeitung. 2010a. Guttenberg sägt an Wehrpflicht. https://www.sueddeutsche.de/politik/ bundeswehr-unter-sparzwang-guttenberg-saegt-an-wehrpflicht-1.957400. Zugegriffen: 11. Okt. 2019.

Süddeutsche Zeitung. 2010b. Wehrpflicht: Seehofer pfeift Guttenberg zurück. https://www.sueddeutsche. de/politik/politik-kompakt-wehrpflicht-seehofer-pfeift-guttenberg-zurueck-1.954322. Zugegriffen: 15. Juli 2020.

Tagesspiegel Online. 2002. Bundeswehr: Dienst ist Dienst. https://www.tagesspiegel.de/politik/bundes wehr-dienst-ist-dienst/303246.html. Zugegriffen: 2. Okt. 2019.

Viehöver, Willy. 2008. Die Wissenschaft und die Wiederverzauberung des sublunaren Raumes. Der Klimadiskurs im Licht der narrativen Diskursanalyse. In Forschungspraxis, 3. Aufl., Handbuch Sozialwissenschaftliche Diskursanalyse, Bd. 2, Hrsg. Reiner Keller, Andreas Hirseland, Werner Schneider, und Willy Viehöver, 233-269. Wiesbaden: VS. 
Viehöver, Willy. 2011. Diskurse als Narrationen. In Theorien und Methoden, 3. Aufl., Handbuch Sozialwissenschaftliche Diskursanalyse, Bd. 1, Hrsg. Reiner Keller, Andreas Hirseland, Werner Schneider, und Willy Viehöver, 193-224. Wiesbaden: VS.

Viehöver, Willy. 2013. Keep on Nano truckin', truck our blues away. Zur Rolle von Sprache und Narrativen in der diskursiven Governance der Wissensproduktion im Feld der Nanotechnologien. In Diskurs - Sprache - Wissen. Interdisziplinäre Beiträge zum Verhältnis von Sprache und Wissen in der Diskursforschung, Hrsg. Willy Viehöver, Reiner Keller, und Werner Schneider, 213-290. Wiesbaden: Springer VS.

Viehöver, Willy. 2014. Erzählungen im Feld der Politik, Politik durch Erzählungen. Überlegungen zur Rolle der Narrationen in den politischen Wissenschaften. In Politische Narrative. Konzepte - AnalysenForschungspraxis, Hrsg. Frank Gadinger, Sebastian Jarzebski, und Taylan Yildiz, 67-91. Wiesbaden: Springer VS.

Welt Online. 2010. Umfrage: Mehrheit für Aussetzen der Wehrpflicht. https://www.welt.de/welt_print/ politik/article8898629/Umfrage-Mehrheit-fuer-Aussetzen-der-Wehrpflicht.html. Zugegriffen: 31. Okt. 2019.

Werkner, Ines-Jacqueline. 2011. Wehrpflicht und Zivildienst - Bestandteile der politischen Kultur? Aus Politik und Zeitgeschichte (APuZ) 61(48):39-45.

Yildiz, Taylan. 2020. Die Politik von Erzählräumen. Ein sprachanalytisch-narratives Modell für die Regierungsforschung. Zeitschrift für Politikwissenschaft (ZPol) 30(1):27-51.

Yildiz, Taylan, Frank Gadinger, und Sebastian Jarzebski. 2015. Das narrative Element des Politischen: Überlegungen zu einer Poetologie des Wissens in der Politikwissenschaft. Zeitschrift für Politikwissenschaft (ZPol) 25(3):421-431.

Yildiz, Taylan, Frank Gadinger, und Christopher Smith. 2018. Narrative Legitimierung: Exekutive, repräsentative und subversive Erzählstrategien in der Überwachungskontroverse. Leviathan 46(1):135-162.

ZEIT Online. 2011a. Bundesrat besiegelt Aussetzung der Wehrpflicht. https://www.zeit.de/news-042011/ 15/iptc-hfk-20110415-66-29848988xml. Zugegriffen: 19. Aug. 2019.

ZEIT Online. 2011b. Die Chronologie der Plagiatsaffäre. https://www.zeit.de/politik/deutschland/201103/guttenberg-chronologie-plagiatsaffaere. Zugegriffen: 7. Okt. 2019. 